\title{
An influence of temperature on reconstructed middle ear with shape memory prosthesis
}

\author{
Rafal Rusinek $\mathbb{D} \cdot$ Krzysztof Kecik $\cdot$ Marcin Szymanski $\cdot$ Joanna Rekas
}

Received: 8 July 2017 / Accepted: 11 November 2017/Published online: 18 November 2017

(C) The Author(s) 2017. This article is an open access publication

\begin{abstract}
In the paper idea of reconstructed middle ear with a prosthesis made of shape memory alloy is proposed. The new design of shape memory prosthesis is used to enable adjusting its length to individual patient's needs which is a novel contribution of the paper. In order to make a proper fit of prosthesis, a surgeon has to adjust its size and position by cutting step by step classical prosthesis. It takes time of a operation and enlarges a period of patient's esthesia. Therefore, a shape memory prosthesis (SMP) is proposed to shorten operation time and improve fitting through precisely selected length. A reconstructed middle ear is modelled as a two degree of freedom system with nonlinear shape memory element. Finding advisable periodic and undesirable a periodic and irregular behaviour in various temperature is the main aim of the paper. Results of the study should give an answer whether SMP can be useful in medical practice and should also explain dynamics of the middle ear with SMP. The properties of the shape memory prosthesis, in the form of helical spring, are described here by a polynomial dependence. Firstly, free vibrations are investigated and equilibrium points, next
\end{abstract}

R. Rusinek $(\bowtie) \cdot$ K. Kecik · J. Rekas

Department of Applied Mechanics, Lublin University of

Technology, Lublin, Poland

e-mail: r.rusinek@pollub.pl

M. Szymanski

Department of Otolaryngology Head and Neck Surgery,

Medical University of Lublin, Lublin, Poland forced vibrations are studied under different parameters of external excitations and temperature range. Bifurcation analysis and stability of periodic solutions are performed in order to reveal the system behaviour. Finally, interesting dynamical findings of chaotic vibrations pure regular and regular oscillations with fluctuation are presented. However, from practical point of view only harmonic response of the stapes is advisable. That can be achieved at the normal temperature of a human body only for small excitation amplitude.

Keywords Middle ear dynamics - Shape memory prosthesis $\cdot$ Reconstructed middle ear

\section{Introduction}

The human middle ear consists of three ossicles: a malleus, an incus and a stapes. The ossicles form a sound conduction system which transmits sound from the external ear to the fluids of the inner ear. The ossicles are connected to each other by the incudomallear and the incudo-stapedial joint. The ossicular chain is supported by two muscles: the tensor tympani muscle attached with its tendon to the handle of the malleus, and the stapedius muscle attached to the stapes neck or posterior crus. The malleus is also firmly connected to the tympanic membrane while stapes is 
attached to the bony walls of the oval window by the annular ligament forming stapediovestibular junction. Such a complex bio-system is modeled in the literature from the last half century. The first study in this field was published in 1961 by Möller [22] where the first scheme of middle ear mechanism was proposed. Next, a similar model was investigated by Zwislocki [42]. In both publications, authors used an electrical circuit to analyse middle ear behaviour. They based on Bárány theory which claims that the ossicles rotate around an axis through the head of the malleus and the short process of the incus. Zwislocki assumed that there is a rigid coupling between malleus and incus, in fact he omitted this joint from the analog circuit. Next stage of middle ear modelling started from 1978 when finite element method (FEM) was used to study spatial vibrations of the tympanic membrane and the ossicles $[1,5,7,12,13,38,39,41]$. Although, FEM is very helpful but its accuracy depends on the effort put in geometric and mechanical properties. Moreover, FEM does not allow full description of system dynamics and parameters influence on the system behaviour. Therefore, in the last decades mechanical multi-body systems are developed where ossicles are represented by lumped masses, connected with springs and dashpots. In the literature, one can find three [26], four [23] and sometimes even six degrees of freedom (dof) models [10]. Generally these models focus only on kinematics of an intact middle ear while dynamics is analysed hardly ever. However, Feng and Gan [10] present dynamics of the model described by differential equations of motion in matrix form. They have found the natural frequencies of such a dynamical system. As a result, displacement of the stapes is compared with experimental outcomes giving quite good agreement but still only for intact ossicular chain. Probably, a low number of dynamic middle ear models arises from very complex procedure of obtaining damping and stiffness coefficients which cannot be found in simple experimental tests. The coefficients have to be determined by fitting procedure to get the model response similar to real ear vibrations. Moreover, in most papers, proposed in the literature, only linear models are considered which cannot explain complexity of middle ear behaviour, specially at higher frequencies which are reported for instance in $[15,16,18]$.

A middle ear disfunction is the separate problem which is common in the medical practice and described in literature. The ossicular chain can be partially destroyed by inflammatory diseases such as chronic suppurative otitis media or cholesteatoma. Then, an ossiculoplasty, or reconstruction of the middle-ear ossicles is necessary to improve hearing process of a patient. For more than 50 years middle ear surgery techniques have enabled to improve hearing destroyed by various diseases. Numerous procedures are currently used in clinical practice and a variety of middle ear prostheses are available. The choice of prosthesis type, its material and size is a main practical problem. Adjustment of a prosthesis to a specific anatomical condition in the middle ear is one of the main difficulties. Taking into account a wide range of variables influencing a perfect ossicular reconstruction there is no single ideal ossicular prosthesis. A classical prostheses Total Ossicular Replacement Prosthesis (TORP) and Partial Ossicular Replacement Prosthesis (PORP) are made of titanium. Their length can be adjusted but only once during operation. If the prosthesis is too short, reuse of it is not possible. Our idea, presented in this paper, consists in developing the 'smart' prosthesis made of modern shape memory alloy (SMA), which would be able to adjust its size and angulation to requirements in a specific medical case. Now, the piston-stapes prostheses (NiTiBOND) made of Nitinol are known in the literature and sold by medical concerns. Clinical tests prove that the acousto-mechanical properties of self-crimping prostheses are better because of better sound transmission properties $[3,14]$. However, to the best of authors knowledge there is no study which rises problems of PORP or TORPP prostheses made of SMA. Modelling of a shape memory effect (SME) is very difficult for the sake of shape memory and pseudoelastic effect. Generally, SMAs are a family of metals with the ability of changing their shape depending on temperature [34]. SMAs undergo thermoelastic phase transformations, which can be induced either by temperature or stress. Thermomechanical properties of SMA can be modeled either by microscopic or macroscopic point of view. The microscopic approach treats phenomena in molecular level while from our point of view macroscopic approach is more interesting because takes into account phenomenological features [24]. There is a class of models known as models with assumed phase transformation kinetics which consider preestablished simple mathematical function to describe phase 
transformation. This approach was first proposed by Tanaka and Nagaki [40]. This work motivated other researchers to present modified transformation kinetics laws, e.g. Liang and Rogers [19], Brinson [2]. These models are probably the most popular in the literature and play important role in modelling [24]. Fremond [11] developed a three dimensional model which is able to reproduce the pseudoelastic and shape memory effects by using three internal variables. Very often a one-dimensional model, built up on the original Fremond's model is developed in many papers $[25,30,31,33,36]$. Relatively simply onedimensional model based on Devonshire's theory was proposed by Falk [8] and Falk and Konopka [9]. This model assumes a polynomial-free energy potential, which allows describe both SME and pseudoelastic behavior. Shape memory effect can be one or twoway. In case of one-way SME there is no reverse change of SMA's shape after subsequent cooling. Two-way SME is characterized by a change of SMA's shape during cooling. For the need of middle ear prosthesis we use Nitinol with one-way SME because the prosthesis should change shape after heating and must not recover back when cooling.

In this paper, a nonlinear unidimensional three degrees of freedom (3dof) model of middle ear is proposed because three lumped masses represent the malleus, the incus and the stapes of the intact middle ear. The model is next rebuild to a 2 dof system with ossicular prosthesis made of shape memory alloy. This is because, usually the incus is removed during surgery operation, therefore the model of the reconstructed middle ear has only two bones: the malleus and the stapes. This is the cause to use 2 dof model here. In the paper polynomial function to describe SMA characteristic is engaged. Theoretical consideration of similar 2dof SMA oscillator with the polynomial dependence are quite popular in literature. Savi and Pacheco [34] analysis numerically free and forced vibration of 1 and 2dof SMA oscillator. They focus mainly on chaotic response. The more detailed analysis of 2dof system is presented in [20] and 1dof system in [21]. The authors investigate some aspects of bifurcation phenomenon. The similar numerical analysis of 1 dof system is shown by Shang and Wang [37]. They investigate the periodic or chaotic motion of the system occurring by changing temperature, damping coefficient and amplitude of exciting force. Recently, some papers are published connected to
SMA systems. For instance, 0-1 test is used to diagnose chaos in 1 and 2dof SMA oscillators. [35], time delay procedure is applied to control chaos [17], dynamics of SMA pendulum is presented in [4].

Despite the fact that 2dof SMA system has been analysed in the literature the configuration and parameters proposed here are different because the model relates directly to the small biomechanical system of the human middle ear. The paper is focused on periodic oscillations forced by the harmonic excitation at different temperatures. We are looking for dynamic effects in the model to be sure that we can avoid nonlinear, irregular effects in the reconstructed human middle ear. Moreover, the analysis of free vibrations and equilibrium states is performed to get possible solutions of the middle ear which is activated not through a harmonic signal but through an impuls (e.g. roar, crash, bang).

The paper is organized as follows: Sect. 2 presents 3dof model of the intact middle ear and explain why the model is reduced to 2dof system with SMA prosthesis. Moreover the model of shape memory prosthesis is discussed. Next in Sect. 3, free vibrations of the reconstructed middle ear are shown and an influence of the prosthesis size on the natural frequencies. Sect. 4 presents the system answer on harmonic excitation. Special attention is devoted to periodic response. Finally, discussion of the results and conclusions are presented in Sect. 5.

\section{Middle ear model}

\subsection{Shape memory oscillator}

The human middle ear mechanism consists of three bones (ossicles) the malleus, the incus and the stapes (the pink parts in Fig. 1) which are connected to one another by joints while ossicles system is connected to the temporal bone by ligaments. That complex biomechanical system can be presented in simplified form as a 3dof model (Fig. 2) where three ossicles: the malleus, the incus, and the stapes are represented by the lumped masses $m_{M}, m_{I}, m_{S}$, respectively. The malleus $\left(m_{M}\right)$ is jointed with a tympanic membrane (TM) by means of the spring $k_{T M}$ and the dashpot $c_{T M}$. An anterior malleal ligament (AML) suspending the malleus is simulated as the spring $k_{A M L}$ and the dashpot $c_{A M L}$. The malleus is connected with the incus by the 


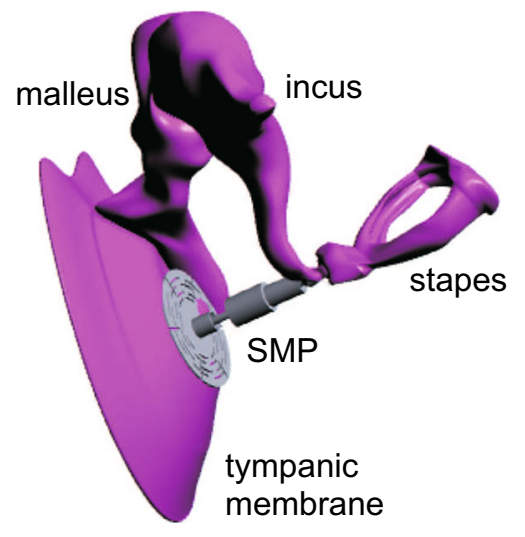

Fig. 1 Intact middle ear (pink parts) and shape memory prosthesis (grey). (Colour figure online)

incudomalleal joint (IMJ) represented by the spring $k_{I M J}$ and the dashpot $c_{I M J}$. Next, the incus and the stapes are supported by the posterior incudal ligament (PIL) and the annular ligament (AL) that are modelled as the springs with stiffness $k_{P I L}$ and $k_{A L}$, and the dashpots $c_{P I L}$ and $c_{A L}$. The incudostapedial joint (ISJ) is shown as the spring $k_{I S J}$ and the dashpot $c_{I S J}$. The stapes motion is transferred to the cochlea (C) which stiffness and damping is expressed by $k_{C}$ and $c_{C}$. Motion of the ossicles is stimulated by sound which approaches to a tympanic membrane (TM) in the form of harmonic excitation $P_{0} \sin \omega t$ where $P_{0}$ and $\omega$ mean the excitation amplitude and frequency.Sometimes, in medical practice, middle ear disfunction happens such as otosclerosis or damage of an ossicular chain, then a hearing process can be improved with the help of ossicular prostheses. In this case, usually an incus is removed and a prosthesis is used to connect the stapes with the tympanic membrane and the malleus (grey part in Fig. 1). Therefore, 2dof system of the intact middle ear is reduced do 2 dof system of the reconstructed ear. Classical prosthesis, commercially available, are made of titanium. They can change its length before a implementation but after that an elongation is not possible. Therefore, a new type of the shape memory prosthesis (SMP) made of Nitinol, which can match its length and shape, depending on activation temperature, to individual patient's needs is proposed. The new SMP (grey part in Fig. 1) consists of the helical spring of $N$ coils and $D$ diameter which is made of the Nitinol wire of the circular cross section (diameter $d$ ). The prosthesis can change its length under the influence of temperature. Since, the incus is usually destroyed in diseased ear, it should be removed. Then, a two degree of freedom (2dof) model of a reconstructed middle ear is proposed where SMP is modelled as the shape memory spring (Fig. 3). The similar model is analysed in [27] and [28]. The first paper ([27]) takes into account the nonlinearity of the annular ligament (AL), which is omitted here to focus only on the temperature effect of SMP. The second ([28]) investigates the problem of soft impact in the middle ear with the shape memory prosthesis.Differential equations of motion of $2 \mathrm{dof}$ model presented in Fig. 3 are written in the form

$$
\begin{aligned}
& m_{M} \ddot{x}_{M}(t)+\left(k_{T M}+k_{A M L}\right) x_{M}+\left(c_{T M}+c_{A M L}\right) \dot{x}_{M}(t) \\
& \quad+c_{S M A}\left(\dot{x}_{M}(t)-\dot{x}_{S}(t)\right)+F_{S M A}=P_{0} \sin \omega t \\
& m_{S} \ddot{x}_{S}(t)+\left(c_{C}+c_{A L}\right) \dot{x}_{S}(t)-c_{S M A}\left(\dot{x}_{M}(t)-\dot{x}_{S}(t)\right) \\
& \quad+\left(k_{A L}+k_{C}\right) x_{S}(t)-F_{S M A}=0
\end{aligned}
$$

$F_{S M A}$ means a longitudinal force of the helical spring of diameter $D$ made of SMA wire (diameter $d$, radius $r$ ). The force is expressed by

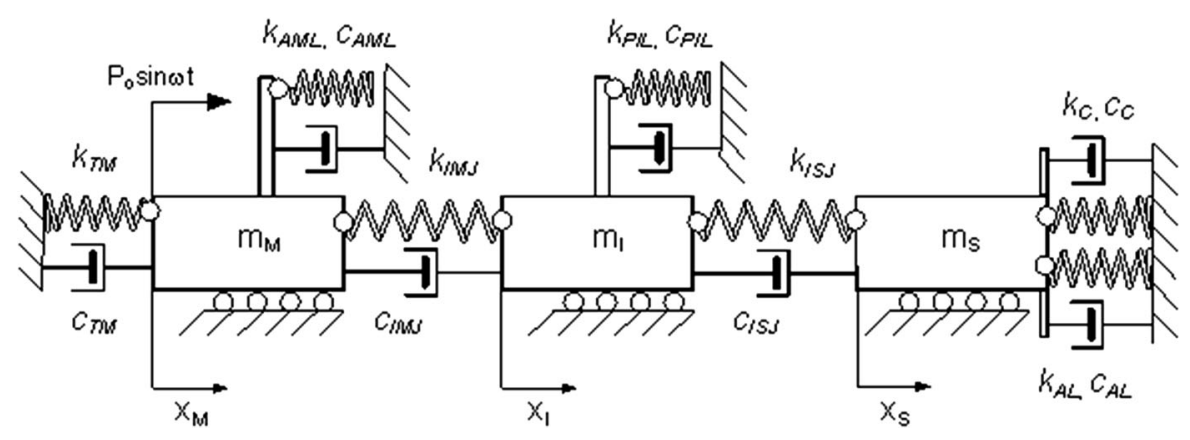

Fig. 2 Three degrees of freedom model of intact middle ear 
Fig. 3 Two degrees of freedom model of reconstructed middle ear with SM helical spring

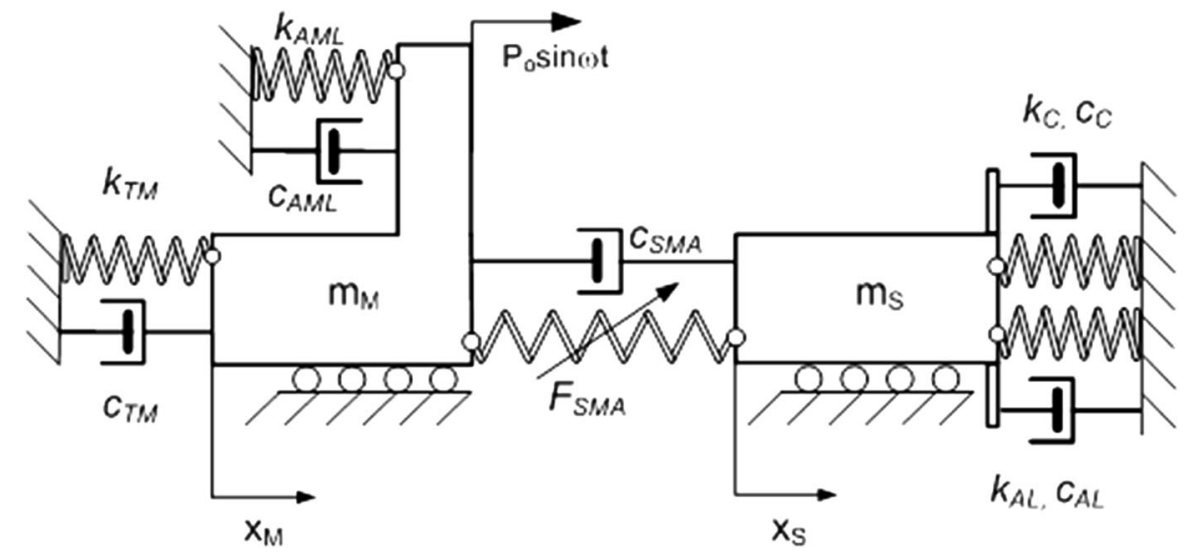

$$
F_{S M A}=\frac{4 \pi}{D} \int_{0}^{d / 2} \sigma r^{2} d r
$$

where, the shear stress $\sigma$ of shape memory element is described by the polynomial function proposed by Falk [8, 32]

$$
\sigma=\frac{\partial W}{\partial \varepsilon}=a\left(T-T_{M}\right) \varepsilon-b \varepsilon^{3}+\frac{b^{2}}{4 a\left(T_{A}-T_{M}\right)} \varepsilon^{5}
$$

The stress dependence (3) is based on Devonshire's theory of a polynomial-free energy $(W)$ which was initially proposed for a one-dimensional media and later extended for three-dimensional context [9].

$$
W(\varepsilon, T)=\frac{a}{2}\left(T-T_{M}\right) \varepsilon^{2}-\frac{b}{4} \varepsilon^{4}+\frac{b^{2}}{24 a\left(T_{A}-T_{M}\right)} \varepsilon^{6}
$$

where $a$ and $b$ are positive material constants, $T_{M}$ and $T_{A}$ means the temperature when martensite (subscript M) and austenite (subscript A) is stable, $\varepsilon$ denotes a strain.

According to this model, neither internal variables nor dissipation potential is necessary to describe pseudoelasticity and SME [24]. Therefore, the only state variables for this model are strain $\varepsilon$ and temperature $T$. Then, the free energy is chosen in such a way that the minima and maxima points represent stability and instability of each phase of the SMA. As it is reported in [24], usually, in one-dimensional models three phases are considered: austenite and two variants of martensite $(\mathrm{M}+, \mathrm{M}-)$. Hence, the free energy is chosen such that for high temperatures ( $T>T_{A}$ ), it has only one minimum at vanishing strain representing the equilibrium of the austenitic phase (A). At low temperatures $\left(T<T_{M}\right)$, martensite is stable, and the free energy must have two minima at nonvanishing strains. At intermediate temperatures $\left(T_{M}<T<T_{A}\right)$, the free energy should have equilibrium points corresponding to both phases. Therefore, the free energy is defined as a sixth-order polynomial equation (Eq. 4).

For helical spring the shear strain $\varepsilon$ is defined as follows

$\varepsilon=\frac{2 r X}{D^{2} N \pi}$

where $X$ means a helical spring deflection, which for the model presented in Fig. 3 is expressed as

$$
X=x_{M}(t)-x_{S}(t)
$$

The restoring force of helical spring is defined by Eq. 2. After integration, the SM spring force $\left(F_{S M A}\right)$ is given as

$$
\begin{aligned}
F_{S M A}= & \frac{4 \pi d^{3}}{D}\left(\frac{a d\left(T-T_{M}\right) X}{32 D^{2} N \pi}-\frac{b d^{3} X^{3}}{48 D^{6} N^{3} \pi^{3}}\right. \\
& \left.+\frac{b^{2} d^{5} X^{5}}{256 a D^{10} N^{5} \pi^{5}\left(T_{A}-T_{M}\right)}\right)
\end{aligned}
$$

Assuming that Eq. 3 is valid for the pure shear stressstrain behaviour and introducing dimensionless time $\tau$, coordinates $x_{1}, x_{2}$ and temperature $\theta$ expressed as follows 

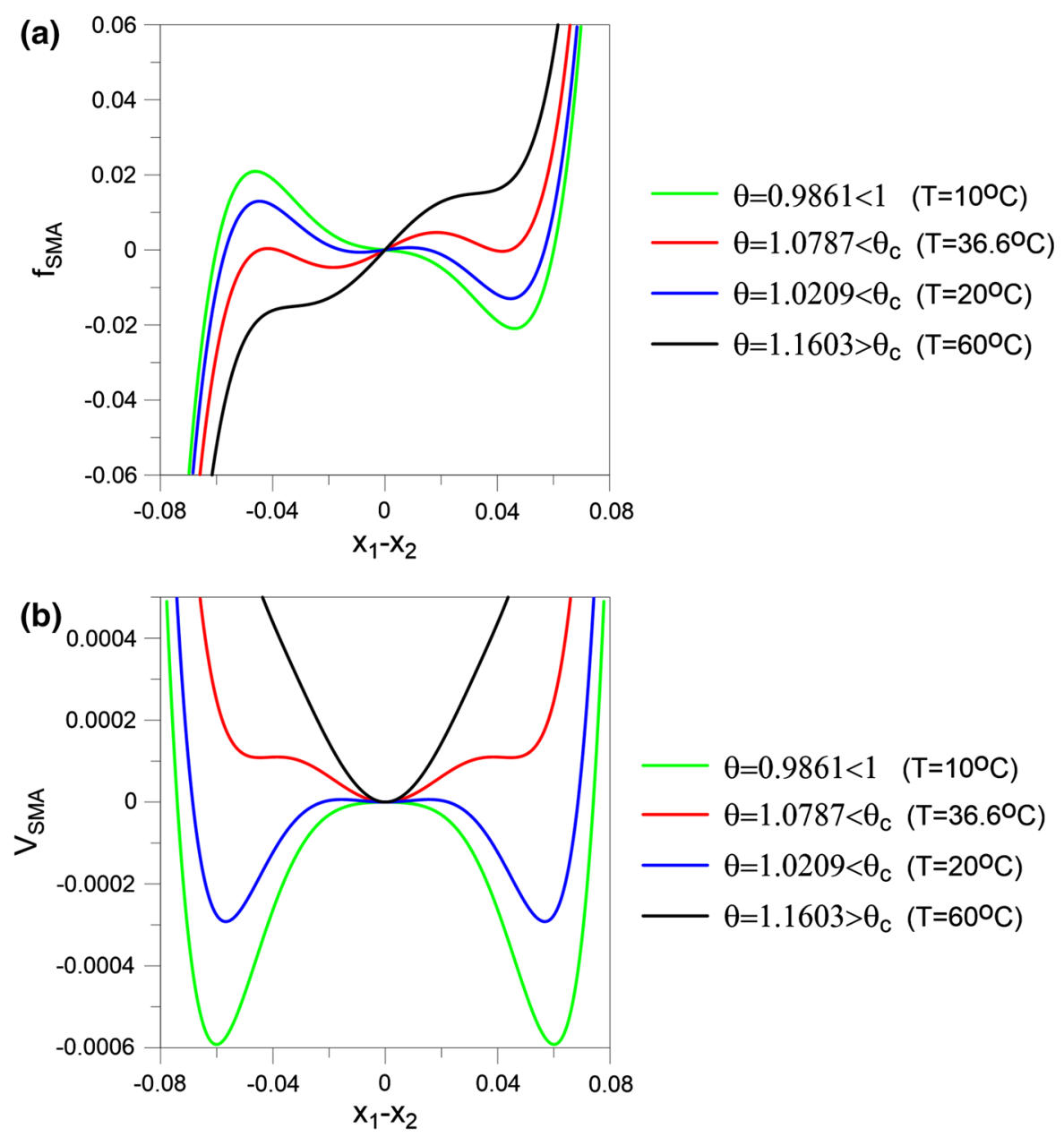

Fig. 4 An influence of temperature on shape memory helical spring. Longitudinal force of SMP (a) and its potential (b)

$$
\tau=\omega_{o} t ; x_{1}=\frac{x_{M}(t)}{x_{s t}} ; x_{2}=\frac{x_{S}(t)}{x_{s t}} ; \theta=\frac{T}{T_{M}} ; x_{s t}=\frac{D^{2} N \pi}{d}
$$

the differential equations of motion (Eq. 1) take nondimensional form

$$
\begin{aligned}
& \ddot{x}_{1}+x_{1} \alpha_{1}+\delta_{1} \dot{x}_{1}+\zeta\left(\dot{x}_{1}-\dot{x}_{2}\right)+f_{S M A}=Q \sin [\tau \Omega] \\
& \ddot{x}_{2}+x_{2} \alpha_{2}+\delta_{2} \dot{x}_{2}-\mu \zeta\left(\dot{x}_{1}-\dot{x}_{2}\right)-\mu f_{S M A}=0
\end{aligned}
$$

where the dimensionless force of SM spring is given as

$$
\begin{aligned}
f_{S M A}= & \beta_{1}(-1+\theta)\left(x_{1}-x_{2}\right)-\beta_{2}\left(x_{1}-x_{2}\right)^{3} \\
& +\beta_{3}\left(x_{1}-x_{2}\right)^{5}
\end{aligned}
$$

The rest of the coefficient are expressed as

$$
\begin{aligned}
& \alpha_{1}=\frac{\left(k_{A M L}+k_{T M}\right)}{m_{M} \omega_{0}^{2}} ; \alpha_{2}=\frac{\left(k_{A L}+k_{C}\right)}{m_{S} \omega_{0}^{2}} ; \beta_{1}=\frac{\pi d^{3} T_{M} a}{8 D m_{M} x_{S t} \omega_{0}^{2}} ; \\
& \beta_{2}=\frac{\pi d^{3} b}{12 D m_{M} x_{s t} \omega_{0}^{2}} ; \beta_{3}=\frac{\pi d^{3} b^{2}}{64 a T_{M} D m_{M} x_{s t} \omega_{0}^{2}\left(\theta_{C}-1\right)} ; \zeta=\frac{c_{S M A}}{m_{M} \omega_{0}} ; \\
& \delta_{1}=\frac{\left(c_{A M L}+c_{T M}\right)}{m_{M} \omega_{0}} ; \delta_{2}=\frac{\left(c_{A L}+c_{C}\right)}{m_{S} \omega_{0}} ; \omega_{0}=\sqrt{\frac{k_{A L}+k_{C}}{m_{S}}} ; \\
& \mu=\frac{m_{M}}{m_{S}} ; Q=\frac{P}{m_{M} x_{s t} \omega_{0}^{2}} ; \theta_{C}=\frac{T_{A}}{T_{M}} ; \theta=\frac{T}{T_{M}}
\end{aligned}
$$

The real parameters of middle ear and SMA are taken form [29] and [24], respectively. Next, the dimensional parameters are recalculated in dimensionless ones, finally receiving $\alpha_{1}=0.0952, \alpha_{2}=1, \beta_{1}=5.0279, \beta_{2}=$ $467.1689, \beta_{3}=134760, \delta_{1}=0.3441, \delta_{2}=0.0455, \zeta=$ $0.1023, \mu=14.0449, \theta_{c}=1.0906$. 

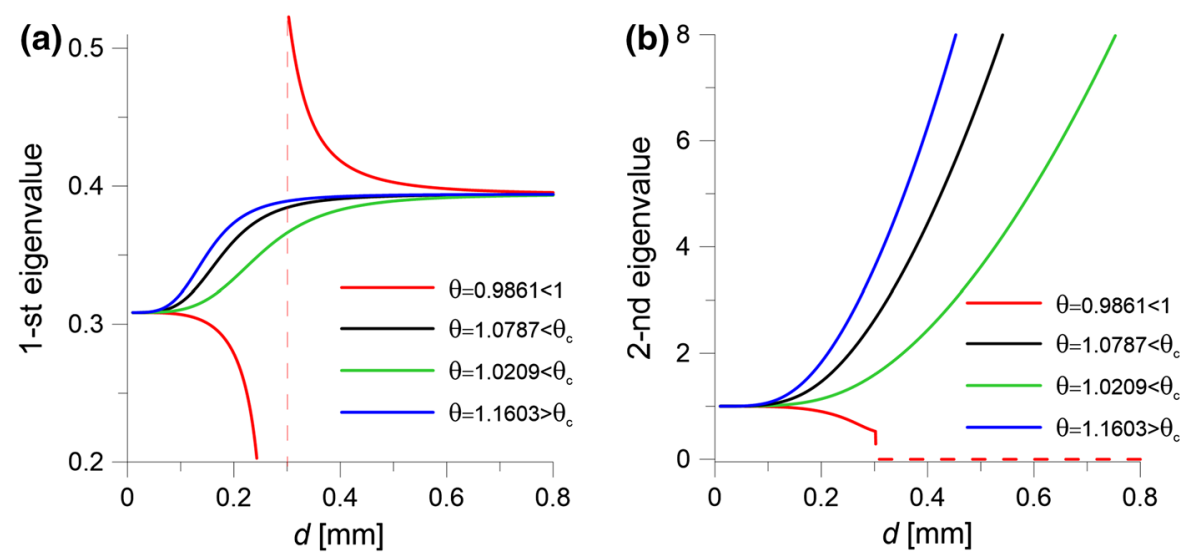

Fig. 5 An influence of the wire diameter $d$ on eigenvalues for various temperature. The first eigenvalue (a), the second one (b)
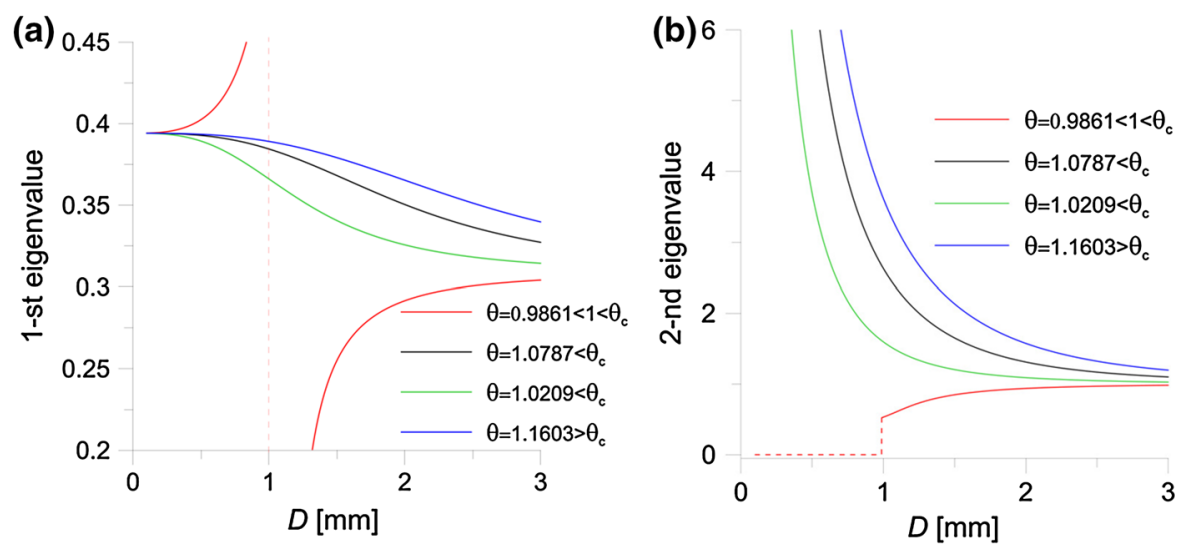

Fig. 6 An influence of the spring diameter $D$ on eigenvalues for various temperature. The first eigenvalue (a), the second one (b)

The restoring force of SM spring $f_{S M A}$ (Eq. 10) depends on spring elongation $\left(x_{1}-x_{2}\right)$ and temperature $(\theta)$ that is illustrated in Fig. 4a. Temperature changes a curve slope that should influences the system behaviour. Additionally, Fig. 4b presents potential of SM spring with characteristic minima and maxima which form a potential wells. When $\theta \leq 1$ the potential has two minima and one maximum. For $1<\theta<\theta_{c}=1.0906$ the potential has three minima, while $\theta>\theta_{c}$ only one minima exists. Potential of $f_{S M A}$ play important role in system's dynamics because the force influences vibrations and equilibriums.

\subsection{Shape memory prosthesis}

The proposed here SMP is made of Nitinol wire (diameter $d=0.2-0.3 \mathrm{~mm}$ ) which has one-way shape memory effect the temperature of austenitic transformation $T_{A} \geq 45^{\circ} \mathrm{C}$. This temperature condition is necessary to activate the prosthesis at temperature which is above the normal human body temperature. $T_{A}$ depends on the nickel to the titanium ratio in SMA. The prosthesis can be formed for example as a helical spring but its diameter cannot exceed $1.2 \mathrm{~mm}$ and length $4 \mathrm{~mm}$ because of space limitation in the human middle ear. The prosthesis has to be taught to increase its length from 4 to $6 \mathrm{~mm}$ after heating above $T_{A}$. Therefore SMP should have 2-4 coils in order to get the proper length. Prosthesis 


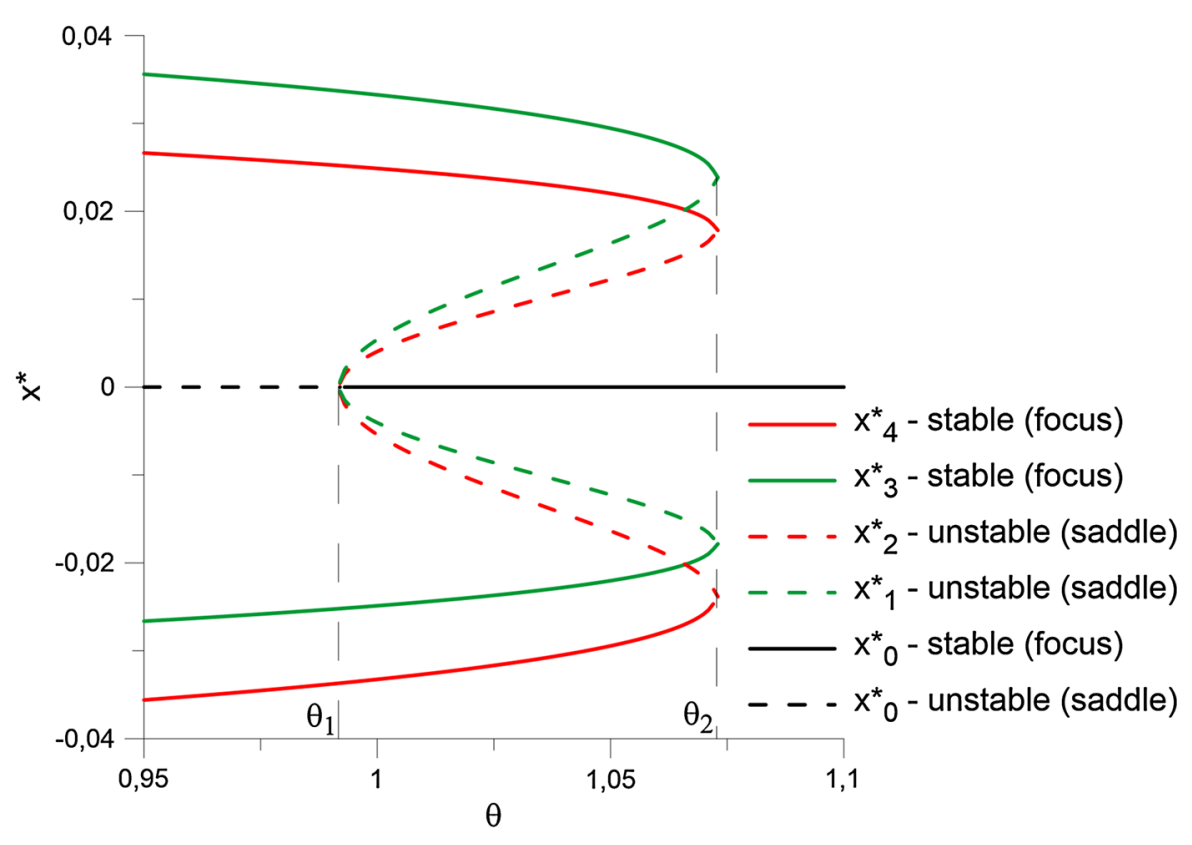

Fig. 7 Temperature influence on system fixed points

implementation during surgery operation is very difficult. At the beginning, SMP in martensite phase is placed between the tympanic membrane and the stapes. Next, the prosthesis is heated very carefully to obtain the austenitic phase and the proper length for individual patient. After cooling to the normal body temperature, the prosthesis length remains the same. Using SMP decreases implementation time and improves the prosthesis fit. For the sake of nonlinear properties of SMA this paper focuses on regular and irregular motions at different temperatures which can be important during implementation. Moreover, it is expected that SMP ensures regular motion of the stapes which next transfer vibration to the inner ear.

\section{Free vibration}

The free response of reconstructed middle ear with SMP is discussed here in two variants. At the beginning, the analysis of simplified linear system is performed in order to calculate natural frequencies of the system and estimate an influence of SMP dimensions. The set of several temperatures in the range from 10 to 60 Celsius degrees is analysed: $T=60^{\circ} \mathrm{C}$ $(\theta=1.1603)$ - the temperature when only austenit phase is stable, $T=36.6^{\circ} \mathrm{C}(\theta=1.0787)$ - the normal temperature of human body, $T=20^{\circ} \mathrm{C}$ $(\theta=1.0209)$ - the usual room temperature and $T=10^{\circ} \mathrm{C}(\theta=0.9861)$ - the temperature when only martensite phase is stable. While, next subsection presents fixed points analysis of the nonlinear system as a function of temperature.

\subsection{Linear vibrations}

From practical point of view a size of SMP is important therefore an analysis of the spring and the wire cross section diameter is perform on simplified linear and undamped system, then $\beta_{2}=0, \beta_{3}=0$, $\delta_{1}=0, \delta_{2}=0, \zeta=0$. This simplified system is analysed in order to estimate analytically an influence of the wire and the spring diameter on the system natural frequencies. Then, the proper prosthesis size could be selected to receive the same natural frequencies like in the intact human middle ear. The mentioned natural frequencies (eigenvalues) of the system depend also on temperature $(\theta)$ as presented in Figs. 5 and 6. First, the wire diameter $(d)$ is analysed. For $\theta>1$ the first eigenvalue (Fig. 5a) grows reaching the value of 0.4 for $d>0.6 \mathrm{~mm}$ while for $\theta<1$ it asymptotically falls down for $d>0.3 \mathrm{~mm}$ next jumps 

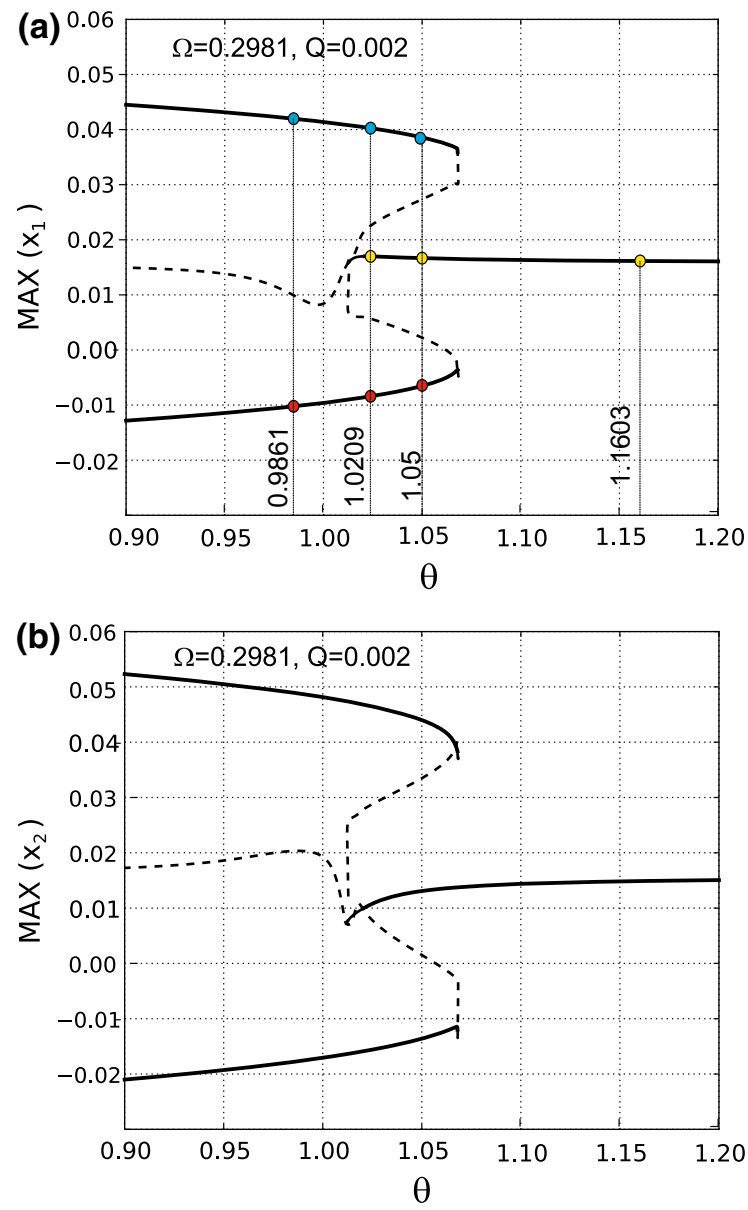

Fig. 8 An influence of temperature on the malleus- $x_{1}$ (a) and the stapes- $x_{2}(\mathbf{b})$ motion

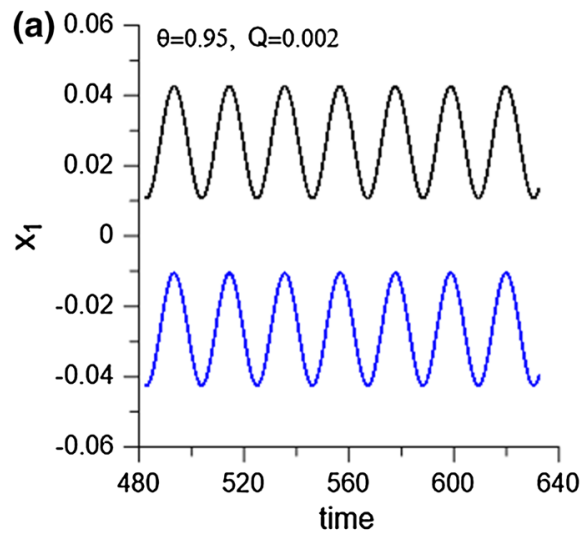

to higher value at $d=0.3 \mathrm{~mm}$ and decreases to 0.4 , the same as for $\theta>1$. Similar behaviour demonstrates the second eigenvalue (Fig. 5b) which increases in case of $\theta>1$, while for For $\theta<1$ the eigenvalue decreases. For $d \geq 0.3 \mathrm{~mm}$ the second eigenvalue does not exist that means a lack of solution.

The same phenomena occur when the spring diameter $D$ increases at fixed $d=0.3 \mathrm{~mm}$ (Fig. 6). For $D=1 \mathrm{~mm}$ and $\theta<1$ the first eigenvalue (Fig. 6a) jumps from big value to smaller while the second exists only for $D>1 \mathrm{~mm}$ (Fig. 6b). In case of $\theta>1$ the first eigenvalue decreases to 0.32 and the second one to 1 .One should be aware that this analysis is proper only for linear system that is for the linear spring without pseudoelasticity, however it tells us about the range of SM spring diameter which can be used to SMP design. Thus, for further analysis the special case is taken into consideration when $D=1 \mathrm{~mm}$ and $d=0.3 \mathrm{~mm}$. For nonlinear system these value does not have to be specific as it was in the full linear system for $\theta<1$.

\subsection{Fixed points of nonlinear system}

In this section, the free response of nonlinear system (reconstructed middle ear with SMP) is discussed. The free response is important from practical point of view because that can tell us about response for an impact caused by roar or bang. The differential equations of motion (Eq. 10) can be rewrite into four differential equations of first order

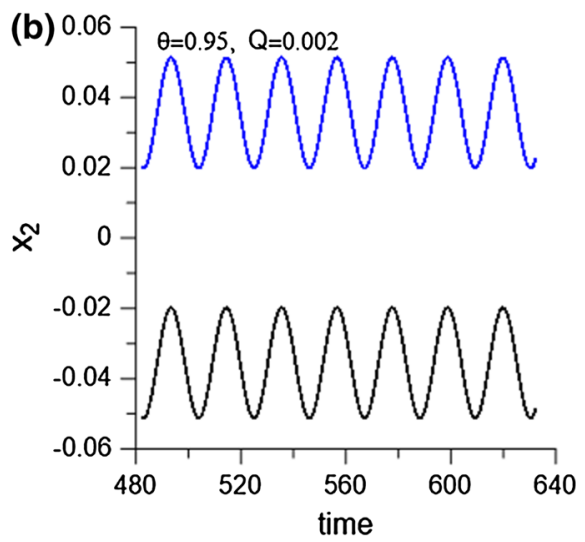

Fig. 9 Time series of the malleus- $x_{1}$ (a) and the stapes- $x_{2}$ (b) obtained for positive initial conditions-black and negative initial conditions-blue. (Colour figure online) 

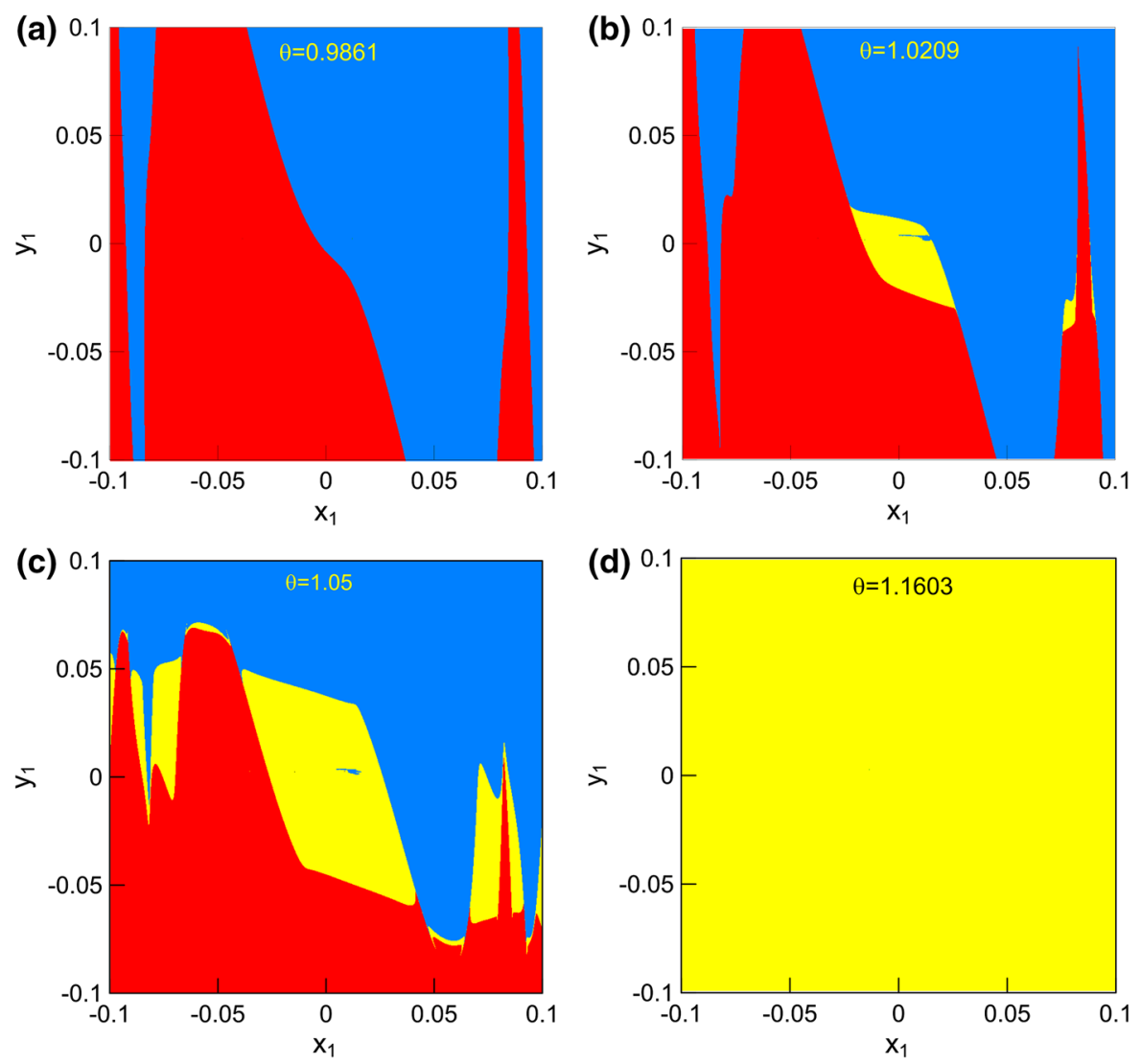

Fig. 10 Basins of attraction for $\theta=0.9861(\mathbf{a}), \theta=1.0209(\mathbf{b}), \theta=1.05(\mathbf{c})$ and $\theta=1.1603$ (d)

$$
\begin{aligned}
& \dot{x}_{1}=y_{1} \\
& \dot{y}_{1}=-\left(x_{1} \alpha_{1}+\delta_{1} y_{1}+\zeta_{1}\left(y_{1}-y_{2}\right)+f_{S M A}\right) \\
& \dot{x}_{2}=y_{2} \\
& \dot{y}_{2}=-\left(x_{2} \alpha_{2}-\zeta_{2}\left(y_{1}-y_{2}\right)+\delta_{2} y_{2}-f_{S M A}\right)
\end{aligned}
$$

The system has different equilibrium points depending on temperature. Denoting by $x_{1}^{*}, y_{1}^{*}, x_{2}^{*}, y_{2}^{*}$, a point that makes the right-hand side of Eq. 13 vanish, the following five possibilities (fixed points) are found

$$
\begin{aligned}
& x_{1}^{*}=0 ; y_{1}^{*}=0 ; x_{2}^{*}=0 ; y_{2}^{*}=0 \\
& x_{1}^{*}=\mp \frac{1}{\sqrt{2}} \sqrt{\frac{\eta_{1} \mp \sqrt{\eta_{2}}}{\beta_{3}\left(\alpha_{2}+\alpha_{1} \mu\right)^{5}}} ;, y_{1}^{*}=0 ; \\
& x_{2}^{*}=-\frac{\alpha_{1} \mu}{\alpha_{2}} x_{1}^{*} ; y_{2}^{*}=0
\end{aligned}
$$

$$
\begin{aligned}
\eta_{1}= & \alpha_{2}^{5} \beta_{2}+3 \alpha_{1} \alpha_{2}^{4} \beta_{2} \mu+3 \alpha_{1}^{2} \alpha_{2}^{3} \beta_{2} \mu^{2}+\alpha_{1}^{3} \alpha_{2}^{2} \beta_{2} \mu^{3} \\
\eta_{2}= & \alpha_{2}^{4}\left(\alpha_{2}+\alpha_{1} \mu\right)^{5}\left(\alpha_{2}\left(\beta_{2}^{2}-4 \beta_{3}\left(\alpha_{1}+\beta_{1}(\theta-1)\right)\right)\right. \\
& \left.+\alpha_{1} \mu\left(\beta_{2}^{2}-4 \beta_{1} \beta_{3}(\theta-1)\right)\right)
\end{aligned}
$$

Among these five possibilities, only those which correspond to real numbers have physical meaning and are presented in Fig. 7. The solid line means stable solution while the dashed line represents unstable one. Stability of these equilibrium is determined by analysis of the Jacobian matrix of Eq. 13 . The trace (Tr) and the determinant (Det) of the Jacobian are defined as follows

where 

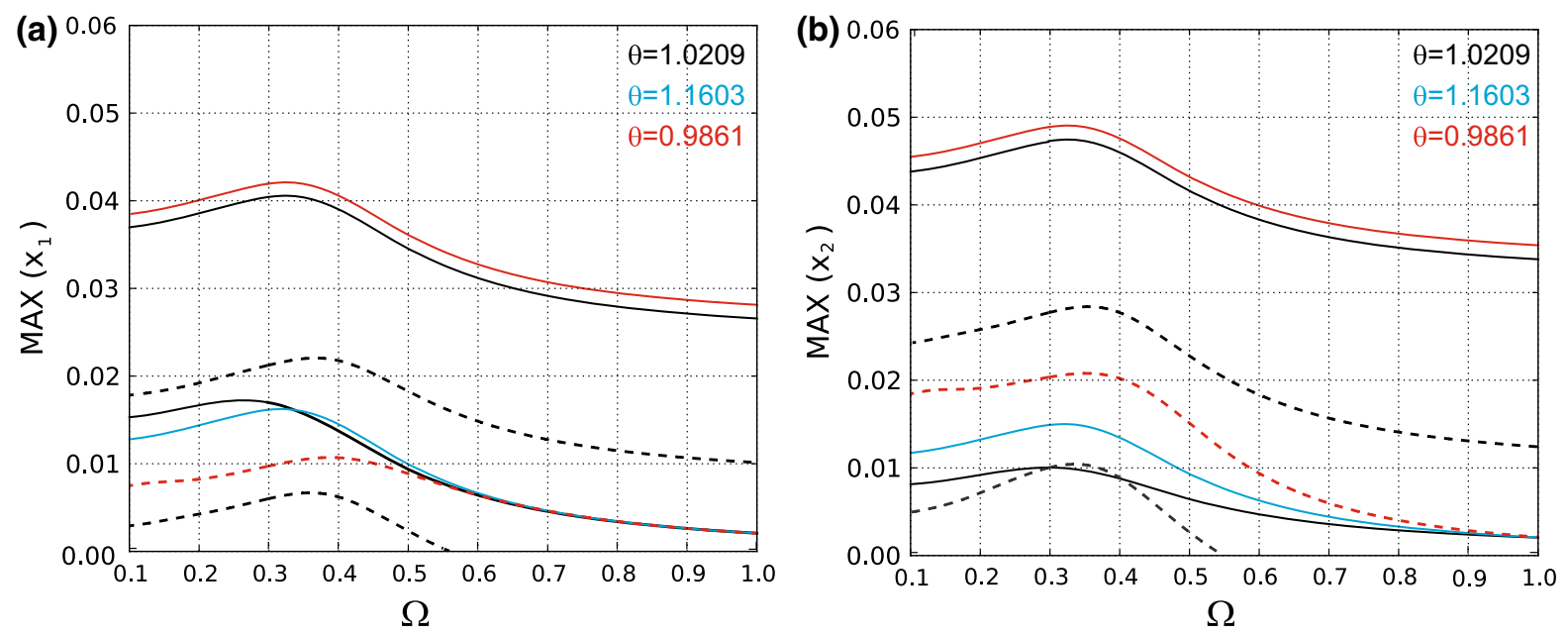

Fig. 11 Resonance curves for the malleus- $x_{1}(\mathbf{a})$ and the stapes- $x_{2}(\mathbf{b})$ at excitation amplitude $Q=0.002$

$$
\begin{aligned}
\operatorname{Tr}(J) & =-\delta_{1}-\delta_{2}-2 \zeta \\
\operatorname{Det}(J) & =\alpha_{1} \alpha_{2}-\alpha_{2} \beta_{1}-\alpha_{1} \beta_{1} \mu+\alpha_{2} \beta_{1} \theta+\alpha_{1} \beta_{1} \theta \mu
\end{aligned}
$$

From the Routh-Hurwitz criterion, the solution can be a saddle (unstable) or focus (stable) as presented in Fig. 7. Therefore, three cases are pointed out depending on $\theta$

- when $\theta<\theta_{1}$ the system has three fixed points, the saddle (unstable trivial solution - gray dashed line) and the other two fixed points which are stable focus (red and green color).

- when $\theta_{1}<\theta<\theta_{2}$ the system has five fixed points, the stable focus (trivial solution - gray color), the other two fixed points are stable focus (red and green color) and the two unstable saddles (blue and black dashed lines).

- when $\theta>\theta_{2}$ the system has only one fixed point, that is the stable focus (trivial solution - gray color).

Thus, the fixed points and their stability depend on regions defined by $\theta_{1}$ and $\theta_{2}$ which result from the analysis of Eq. 13 and are given as follows

$$
\begin{aligned}
& \theta_{1}=\frac{-\alpha_{1} \alpha_{2}+\beta_{1}\left(\alpha_{1} \mu+\alpha_{2}\right)}{\beta_{1}\left(\alpha_{1} \mu+\alpha_{2}\right)} \\
& \theta_{2}=\frac{\beta_{2}^{2}\left(\alpha_{2}+\alpha_{1} \mu\right)-4 \beta_{3}\left(\alpha_{1} \alpha_{2}-\alpha_{2} \beta_{1}-\alpha_{1} \beta_{1} \mu\right)}{4 \beta_{1} \beta_{3}\left(\alpha_{2}+\alpha_{1} \mu\right.}
\end{aligned}
$$

From practical point of view the stable trivial solution is desirable as a free response on the impact produced by a bang or a roar. Therefore, SMP should work at temperature $\theta>\theta_{2}$ because then the system has the only one point of stable equilibrium.

\section{Excited vibrations}

Human speech is composed of a lot of harmonic signals-polyharmonics. Therefore, in a real life a human ear is usually forced harmonically or polyharmonically when speaking and listening to. Insomuch as, in the analysis of excited vibrations, we focus on the frequency which is near to the first natural frequency of the human ear. This frequency is responsible for speech recognition. Therefore, in this section a dynamic response of the ossicles to harmonic excitation is presented under various force amplitudes and temperature conditions near the first resonance which is the most important from practical point of view. In the subsequent sections a possibility of periodic and different kind of aperiodic including irregular vibrations is analysed.

\subsection{Periodic vibrations}

According to the current knowledge, vibrations of the intact middle ear are periodic, therefore the response of reconstructed ossicular chain must be regular as well. Any aperiodicity can disturb sound transmission 

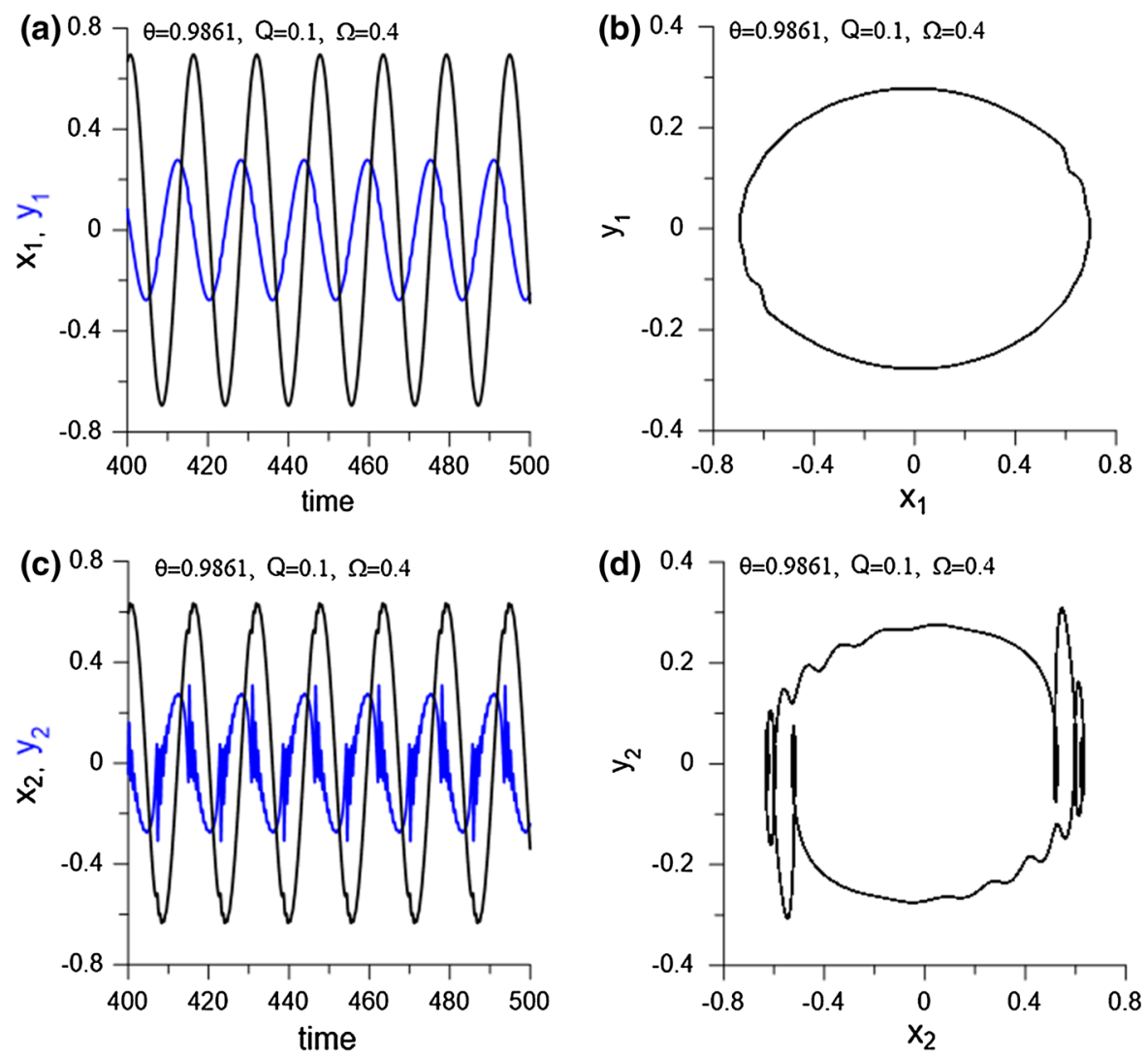

Fig. 12 Time series a, $\mathbf{c}$ of displacements $\left(x_{1}\right.$ and $\left.x_{2}\right)$, velocities $\left(y_{1}\right.$ and $\left.y_{2}\right)$ and trajectories on phase space $\mathbf{b}$, d obtained for $\theta=0.9861, Q=0.1, \Omega=0.4$.

from an outer to an inner ear. In this section analysis of periodic solutions is performed with the help of continuation method (AUTO-07P, [6] ) and next verified in Matlab and Dynamics using Runge-Kutta algorithm of integration with a relative tolerance of $10^{-6}$. Motion of the malleus $\left(x_{1}\right)$ and the stapes $\left(x_{2}\right)$ in the reconstructed middle ear with SMP is analysed versus relative temperature $(\theta)$ in Fig. 8. Three characteristic regions can exist here, where a stable (solid line) and an unstable (dashed line) periodic solutions are possible. When

- $\theta<1.02$ the system has the two stable symmetric solutions and one unstable,

- when $1.02<\theta<1.07$ the system has two stable (symmetric) and two unstable periodic solutions,

- when $\theta>1.07$ the system has only one stable solution.
If the system has two stable periodic solutions they are always symmetric. This means they have the same amplitude but the one is shifted positive and the second negative (black and blue colour in Fig. 9). Positive and negative solution depends on initial conditions, that is clearly visible on the basins of attraction depicted in Fig. 10. For $\theta=0.9861$ only two solutions exist in the blue and red region of initial conditions (Fig. 10a). Next for $\theta=1.0209$ (Fig. 10b) the third solution in the yellow region appears. This yellow region enlarges with temperature $\theta$ (Fig. 10c). The blue and red regions completely disappear at $\theta>1.07$ (Fig. 10d). Temperature influences resonance curves as well. The curves obtained for excitation $Q=0.002$ and various temperature $(\theta)$, are shown in Fig. 11. The first resonance is about 0.34 . Both coordinates $\left(x_{1}\right.$ and $\left.x_{2}\right)$ demonstrate stable (solid lines) and unstable (dashed lines) solutions. The vibrations amplitude of stable solutions increases with temperature. At normal temperature of a human body 

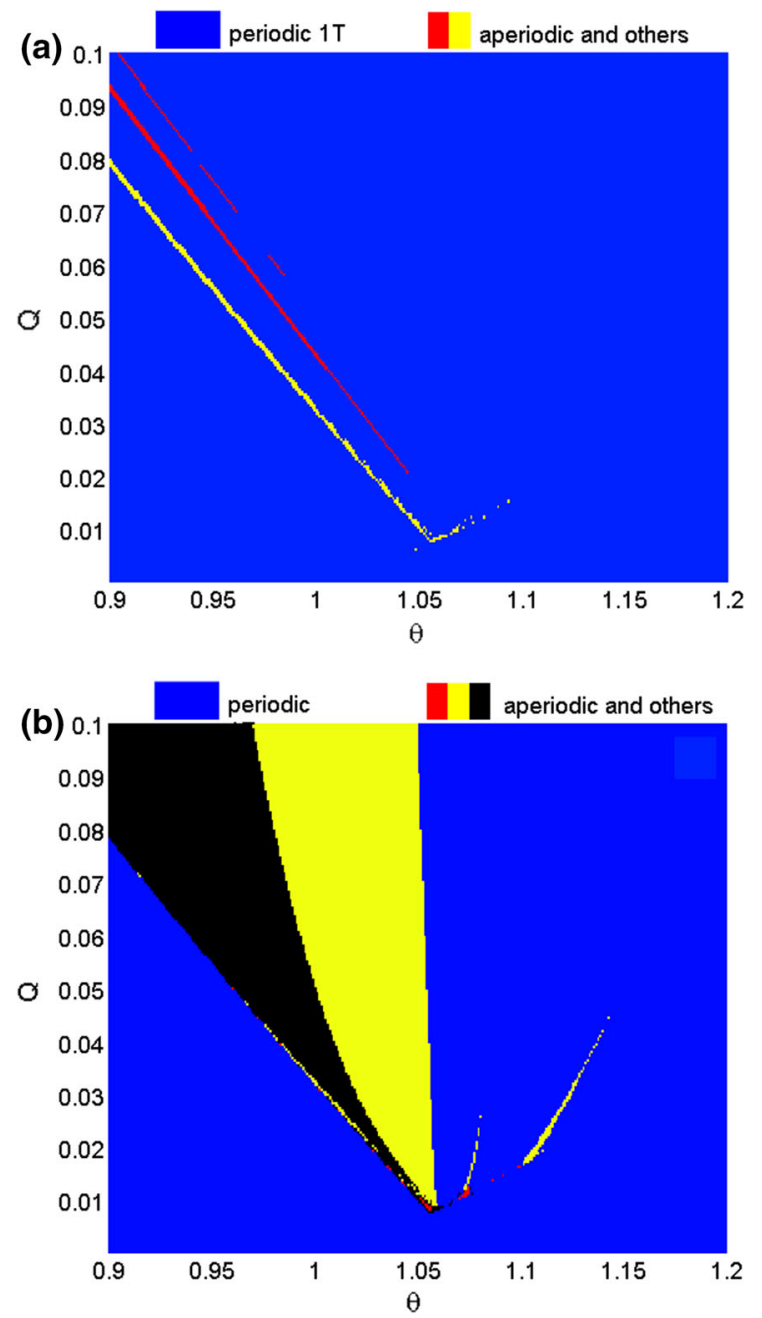

Fig. 13 An influence of excitation amplitude $(Q)$ and temperature $(\theta)$ on the malleus vibrations- $x_{1}$ (a) and velocity- $y_{1}(\mathbf{b})$. The colour represents a various kind of periodic and aperiodic solution. (Colour figure online)

$(\theta=1.0787)$ and also at temperature of SMP activation $(\theta=1.1603)$, the middle ear system demonstrates only one periodic solution (for presented parameters). That is positive aspect from practical point of view. However, besides periodic response the reconstructed middle ear system demonstrates aperiodic and irregular motion which is discussed in the next subsection.

\subsection{Aperiodic vibrations}

Since, the analysed system is nonlinear a probability of aperiodic and even irregular (chaotic) motion is quite big, especially for strong excitation amplitude
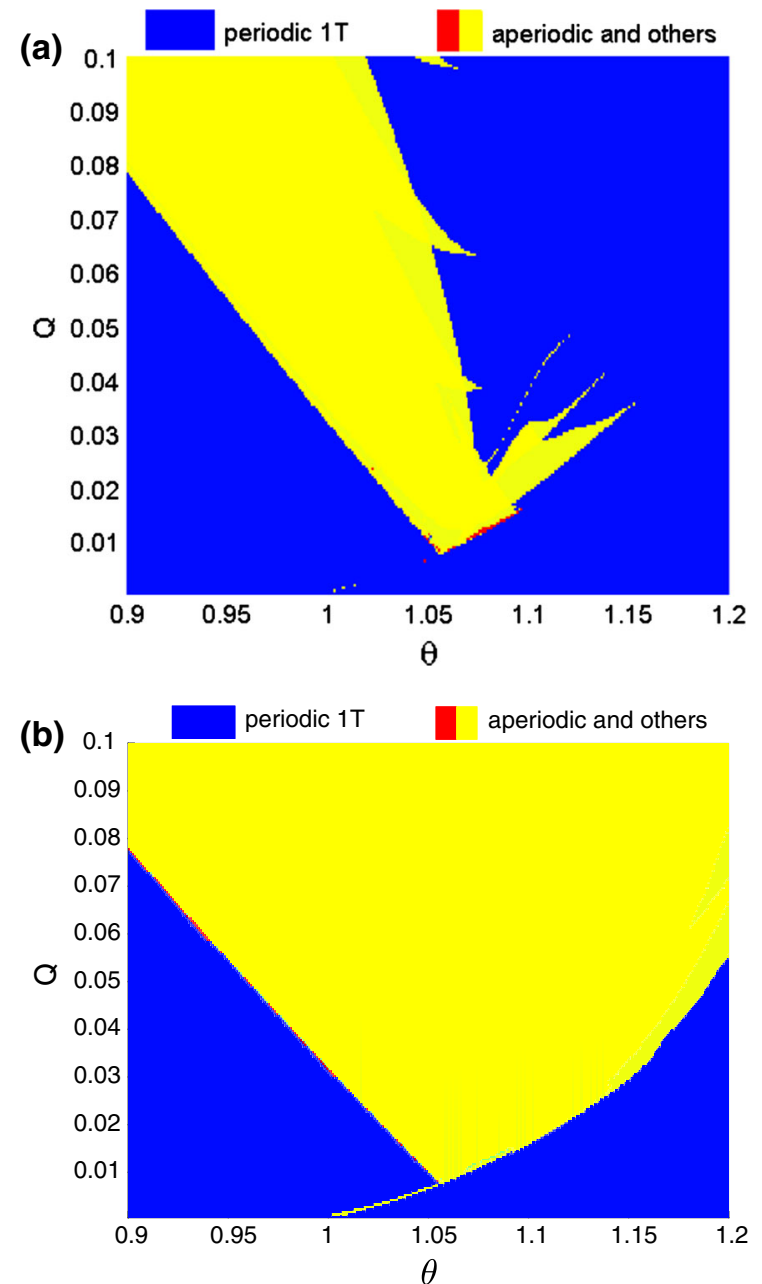

Fig. 14 An influence of excitation amplitude $(Q)$ ana temperature $(\theta)$ on the stapes vibrations- $x_{2}$ (a) and velocity- $y_{2}(\mathbf{b})$. The colour represents a various kind of periodic and aperiodic solution. (Colour figure online)

$(Q)$ at specific temperature $(\theta)$. Therefore, at the beginning an influence of these parameters on periodicity of the ossicles motion is tested and presented in the form of colour maps (Figs. 13, 14). Aperiodicity in this system is fine and difficult to discover because the system can have periodic displacement and aperiodic velocity. An observation of velocity is better because any disturbances of periodicity are visible in velocities first. (see for example the time series and the phase trajectories in Fig. 12). Generally, the response of the reconstructed middle ear system to a periodic excitation is usually harmonic but sometimes with disturbances as presented in Fig. 12 where the family of system aperiodic response obtained for $\theta=$ 

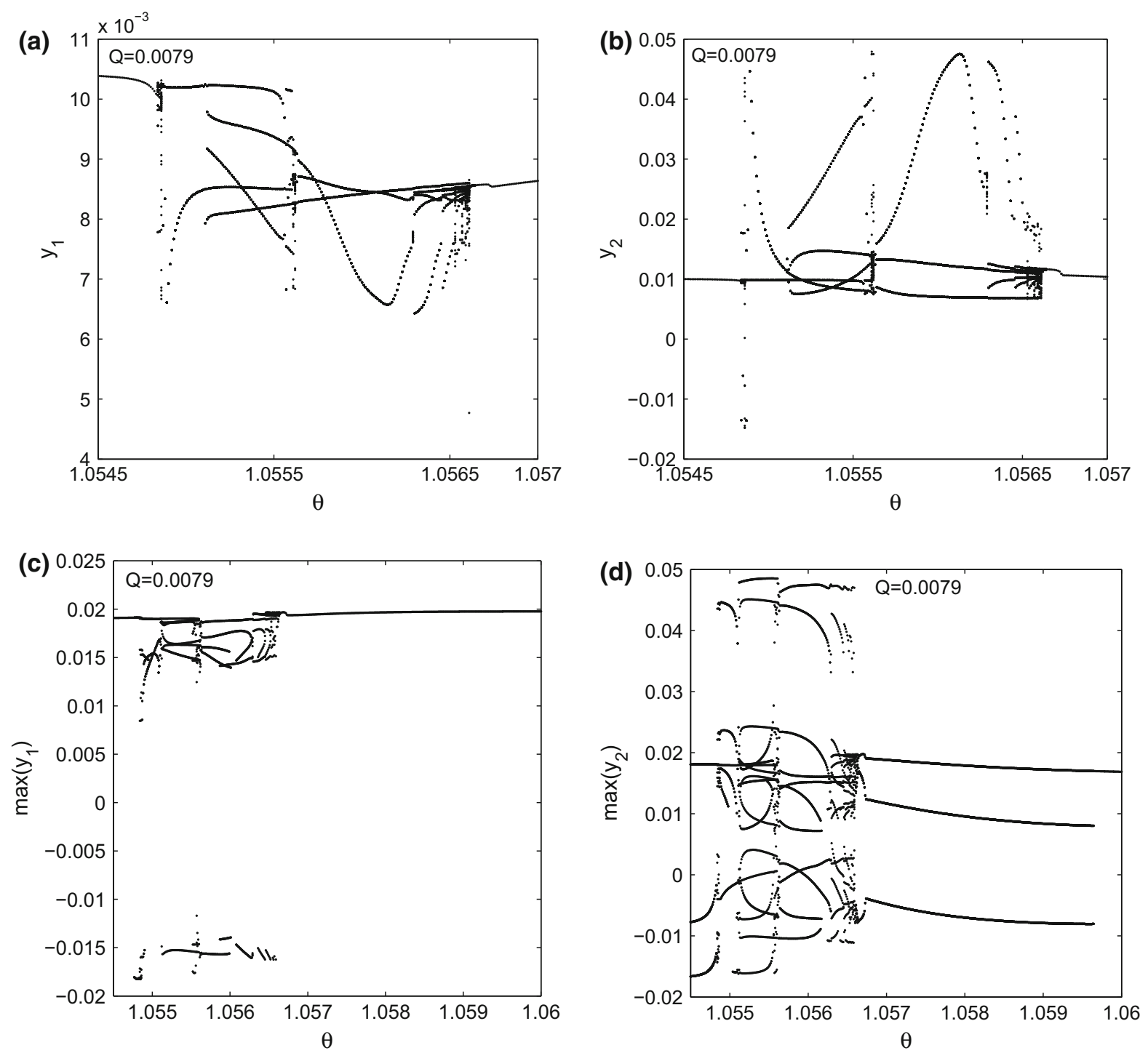

Fig. 15 Classical bifurcation diagrams of the malleus $y_{1}$ (a) and the stapes $y_{2}$ (b) versus temperature $\theta$ at excitation amplitude $Q=0.0079$. Maximums of the malleus (c) and the stapes (d) velocity in one period of excitation

$0.9861, Q=0.1$ and $\Omega=0.4$. is shown. A seeming periodic motion (see $x_{1}$ in Fig. 12a) reveals as aperiodic in the phase space because of small disturbance in velocity. Moreover, different system response is observed for the malleus $x_{1}$ and the stapes $x_{2}$. Since, both the state variables ( $x$ and $y$ ) must be analysed to discover any disturbances of periodic motion, therefore 2-dimensional maps of regularity are presented in Figs. 13 and 14 where displacements ( $x_{1}$ and $x_{2}$, Figs. 13a, 14a) and velocities ( $y_{1}$ and $y_{2}$, Figs. 13b, 14b) are analysed as a function of excitation amplitude $Q$ and temperature $(\theta)$. The blue region
(Figs. 13, 14) means (regular) harmonic solution with period $1 \mathrm{~T}$, then the period of the system response is the same as the period of excitation. Such a typical regular response is thoroughly studied in the previous section. Here, aperiodic and also periodic vibrations but with disturbances (visible especially on velocities) are observed in Figs. 13 and 14 in case of stronger excitation. The yellow, red and black colour mean the region of aperiodic response of different kind and also sub or super-harmonic. Moreover, motion can be harmonic with perturbations in velocities or displacements. On the other hand, there are regions where the 

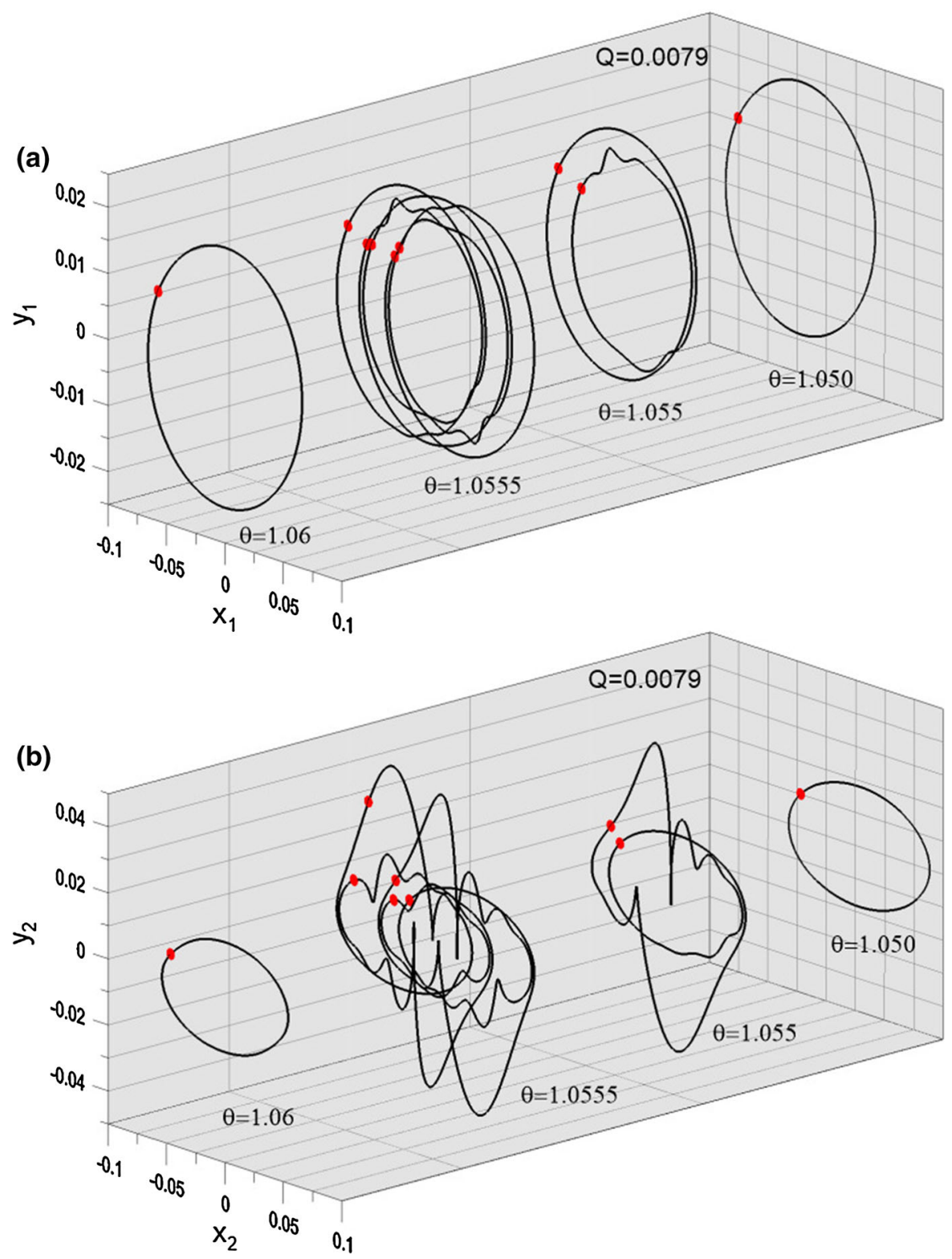

Fig. 16 Change of the malleus (a) and the stapes (b) phase trajectory versus temperature $(\theta)$ at excitation amplitude $Q=0.0079$

malleus motion (displacement $x_{1}$ or velocity $y_{1}$ ) is periodic while the stapes motion $\left(x_{2}\right.$ or $\left.y_{2}\right)$ is aperiodic at the same time. What is more, the malleus and the stapes velocity $\left(y_{1}, y_{2}\right)$ can be aperiodic while the displacement $\left(x_{1}\right.$ and $\left.x_{2}\right)$ is still free of aperiodic oscillations. Generally, the region of aperiodicity increases with external excitation amplitude $Q$.

More information about dynamics can be delivered by the bifurcation analysis which is performed here at different value of excitation amplitude $Q$ to reveal irregular motion, sub-harmonic or harmonic vibrations with any fluctuations. The response of the reconstructed middle ear system is presented in form of the classical bifurcation diagrams of the malleus and the stapes velocity $\left(y_{1}\right.$ and $\left.y_{2}\right)$ collected as a Poincaré points (Figs. 15a, b, 17a, b, 19a, b). Additionally, the diagrams where all the velocity maximums in the time of one period are drawn in Figs. 15c, $\mathrm{d}, 17 \mathrm{c}-\mathrm{f}, 19 \mathrm{c}, \mathrm{d}$. Moreover, for chosen temperatures $(\theta)$ the phase trajectory with red Poincare points are 
plotted (Figs. 16, 18, 20). Usually, in real (life) conditions an excitation of a human ear $Q$ do not exceed 0.02 . Therefore, at the beginning the system response for small excitation $(Q=0.0079)$ is presented as bifurcation diagram in Fig. 15 and the phase trajectory with Poincaré points in Fig. 16. The middle ear system with SMP is very sensitive for temperature $\theta$. At $\theta<1.0547$ (Fig. 15) the ossicles vibrate harmonically with period $1 \mathrm{~T}$ (see also the phase diagram for $\theta=1.050$ in Fig. 16). When $\theta=1.05485$ (Fig. 15) the system vibrates chaotically and next periodically with the period $2 \mathrm{~T}$ (Fig. 16, phase trajectory for $\theta=1.0550$ ) and $5 \mathrm{~T}$ (Fig. 16, phase trajectory for $\theta=1.0555)$. Finally, motion of period $5 \mathrm{~T}$ goes on to the harmonic period 1T (Fig. 16, phase trajectory for $\theta=1.06$ ) through period 3T vibrations. According to the phase trajectories (Fig. 16), the malleus and specially the stapes have additional fluctuations.. In case of stronger excitation $Q=0.02$ the malleus vibrates harmonically (period 1T) in the analysed range of $\theta=0.9-1.2$ with the exception of narrow region of yellow and black colour (Fig. 13). The range of aperiodic motion is wider looking at velocity (Fig. 13b). As usually, the stapes demonstrates much bigger range of aperiodicity than the malleus (Fig. 14). Similarly as previously, there is a small region where motion with period $3 \mathrm{~T}$ and $5 \mathrm{~T}$ is possible (the classical bifurcation diagrams, Fig. 17 and the phase trajectory in Fig. 18 for $\theta=1.028$ and $\theta=1.0295$, respectively). Moreover, chaotic vibrations are also present near $\theta=1.0297$ (Fig. 18). In order to observe ossicles dynamics better, a zoom of Fig. $17 \mathrm{c}, \mathrm{d}$ is presented in Fig. 17d, e. Fortunately, the region of chaotic motion is narrow, near $\theta=1.027$ and $\theta=1.0297$ however, the aperiodicity range of the malleus spreads to $\theta=1.06$ while the stapes motion is aperiodic till $\theta=1.12$ (Fig. 17c, d). Then, motion of the system response demonstrates fluctuations although, the vibration period is 1T starting form $\theta=$ 1.0299 (Fig. 17, and phase portrait in Fig. 18 for $\theta=1.03)$..Strong excitation, expressed by $Q=0.1$ changes system dynamics thoroughly. First of all, the system response is only periodic, both the malleus and the stapes demonstrate vibrations with the period $1 \mathrm{~T}$ (Fig. 19a, b, Fig. 20). However, for the presented model perturbations are present from $\theta=0.9$ (the initial temperature in analysis) to $\theta=1.05$ in case od the malleus and to $\theta=1.27$ for the stapes. As usually, perturbations of the stapes motion are bigger. To sum
Fig. 17 Classical bifurcation diagrams of the malleus $y_{1}$ (a) and the stapes $y_{2}$ (b) versus temperature $\theta$ at excitation amplitude $Q=0.02$. Maximums of the malleus (c, e) and the stapes (d, f) velocity in one period of excitation

up, stronger excitation synchronizes the middle ear response with excitation but does not eliminate perturbations and moreover generates bigger vibrations, what is a typical phenomenon.

\section{Discussion and conclusions}

The concept of shape memory prosthesis is developed here to improve surgery conditions and ensure better prosthesis fitting. That all shorten esthesia time, which is harmful for human being.

Dynamics of the middle ear system with shape memory prosthesis is analysed here in different variants starting from linear vibrations. Natural frequencies of the linear system depend on prosthesis size (diameter) and its temperature. When the relative temperature $\theta<1$ (it means $T<T_{M}$ ) the natural frequencies are not defined unequivocally at the specific wire and spring diameter $(d \approx 0.3 \mathrm{~mm}$ and $D \approx 1 \mathrm{~mm}$, respectively). They have discontinuity observed in Figs. 5 and 6. At these specific points, the middle ear model is not a vibrating system. From practical point of view the temperature of prosthesis at normal work in middle ear is exactly $36.6{ }^{\circ} \mathrm{C}$ then $1<\theta<\theta_{c}$ in this case the effect of vibrations lack does not occur. The analysis of fixed points shows meaningful influence of temperature on fixed points. The system can have 1,3 or 5 fixed points which can be stable or not, but at the normal temperature of human body only one 'zero' equilibrium is stable. This is of special practical importance. More often middle ear is exposed to external excitation then it is expected the ossicles motion should be periodic. Periodic vibrations occur in the all temperature range but in the case of lower temperatures two symmetric solutions appear. Fortunately, near the temperature of human body only one solution is possible but the solution demonstrates smaller vibrations than in the case of lower temperatures. From practical application point of view a change of the prosthesis temperature is possible only during prosthesis implementation and activation. After that the temperature is constant that should 

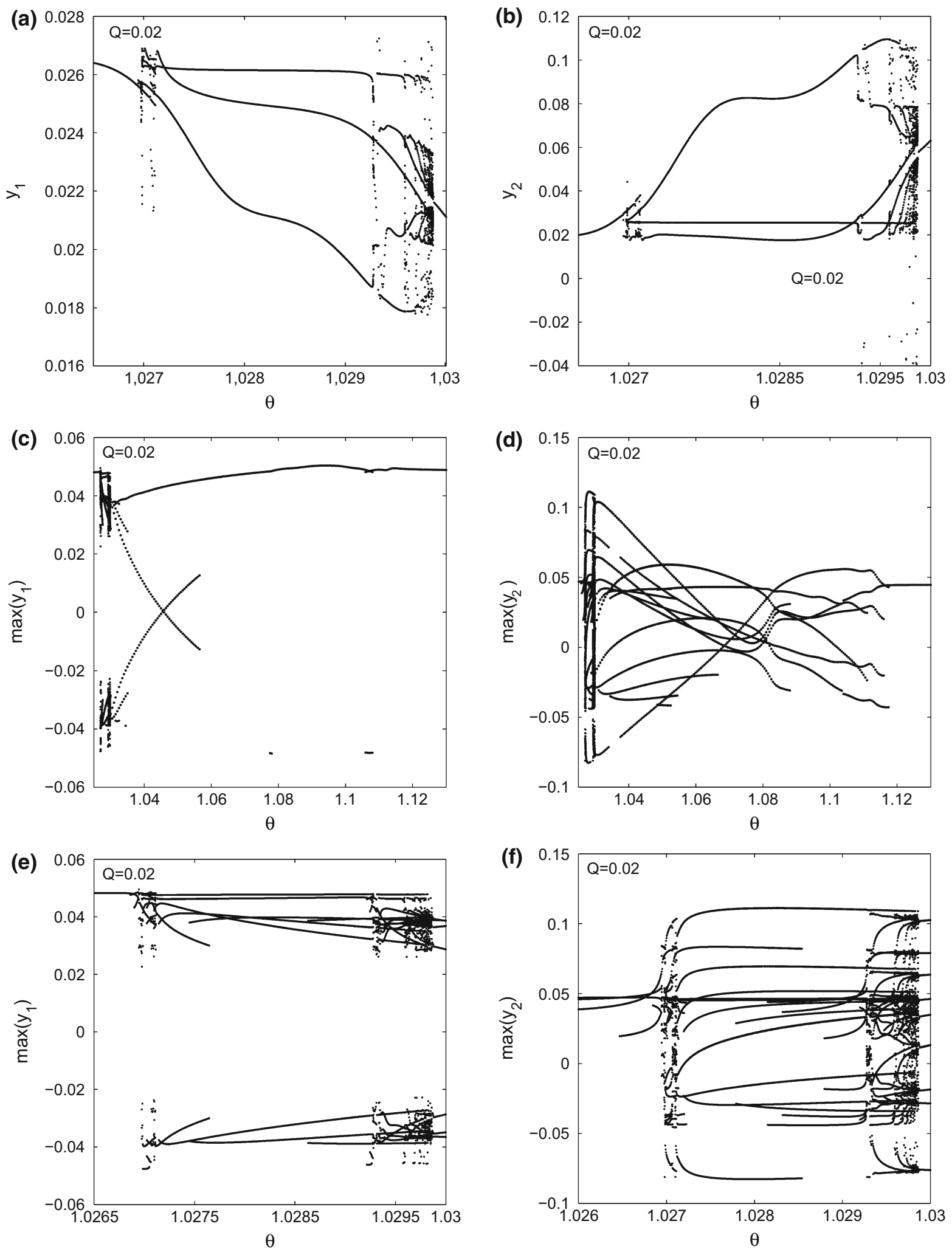
Fig. 18 Change of the malleus (a) and the stapes (b) phase trajectory with temperature $(\theta)$ for $Q=0.02$
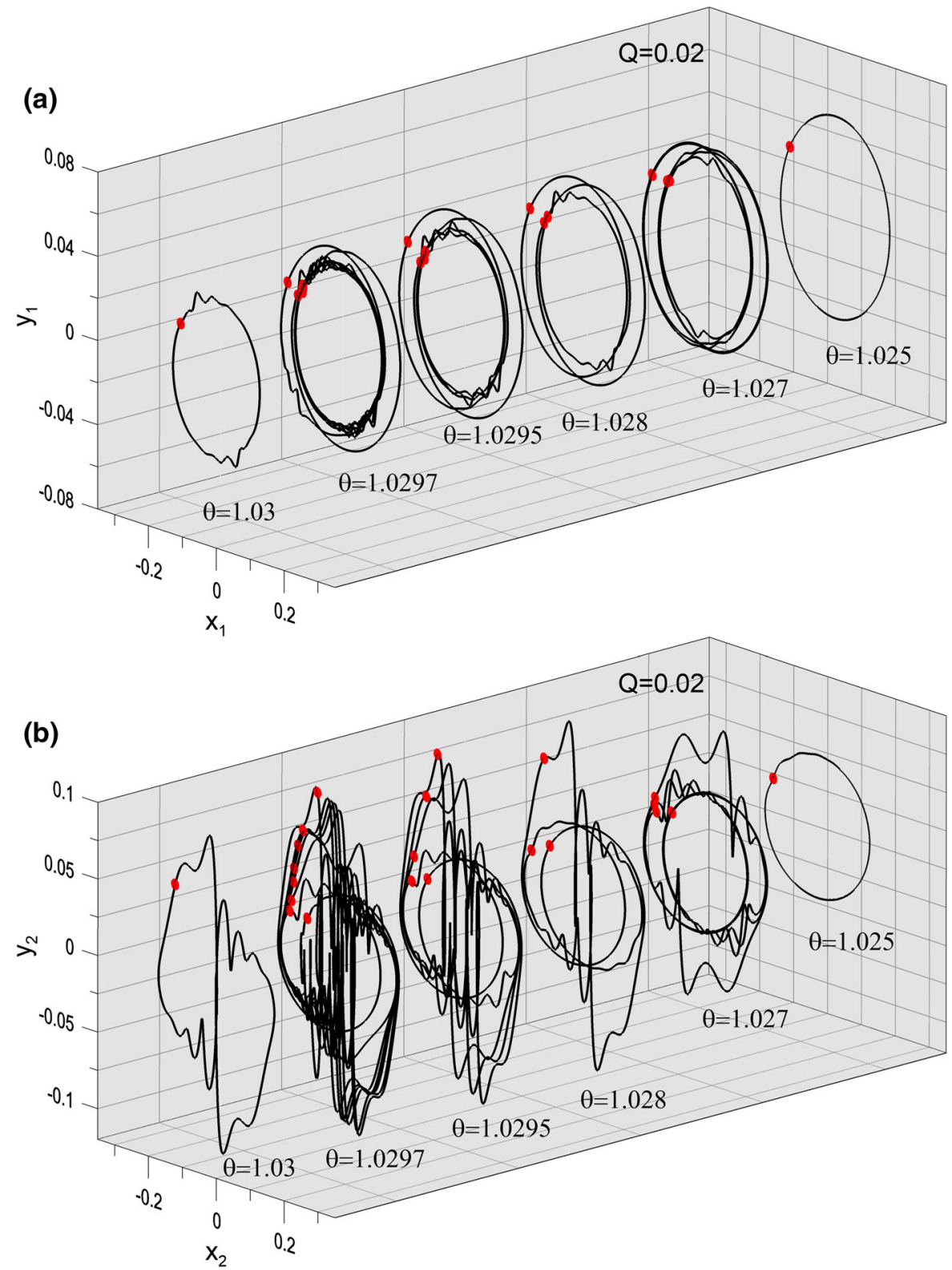

ensure stable working. However, one should remember the ear with SMP is also sensitive to initial conditions.

Unfortunately, the ear with shape memory prosthesis demonstrates aperiodic vibrations as well. The most often, it manifest itself by perturbations which are added to periodic motion. The perturbations are caused by strong nonlinear characteristic of shape memory element (prosthesis). The region of aperiodicity is wider for big excitation amplitude, moreover the stapes shows much bigger aperiodicity range than the malleus. Besides motion with perturbations, the ossicles can vibrate chaotically in narrow range of temperature. The temperature where chaotic motion exists is lower than the normal temperature of human body. Thus, perturbations of periodic motion are only danger when putting shape memory prosthesis to the middle ear during surgery operation. This 

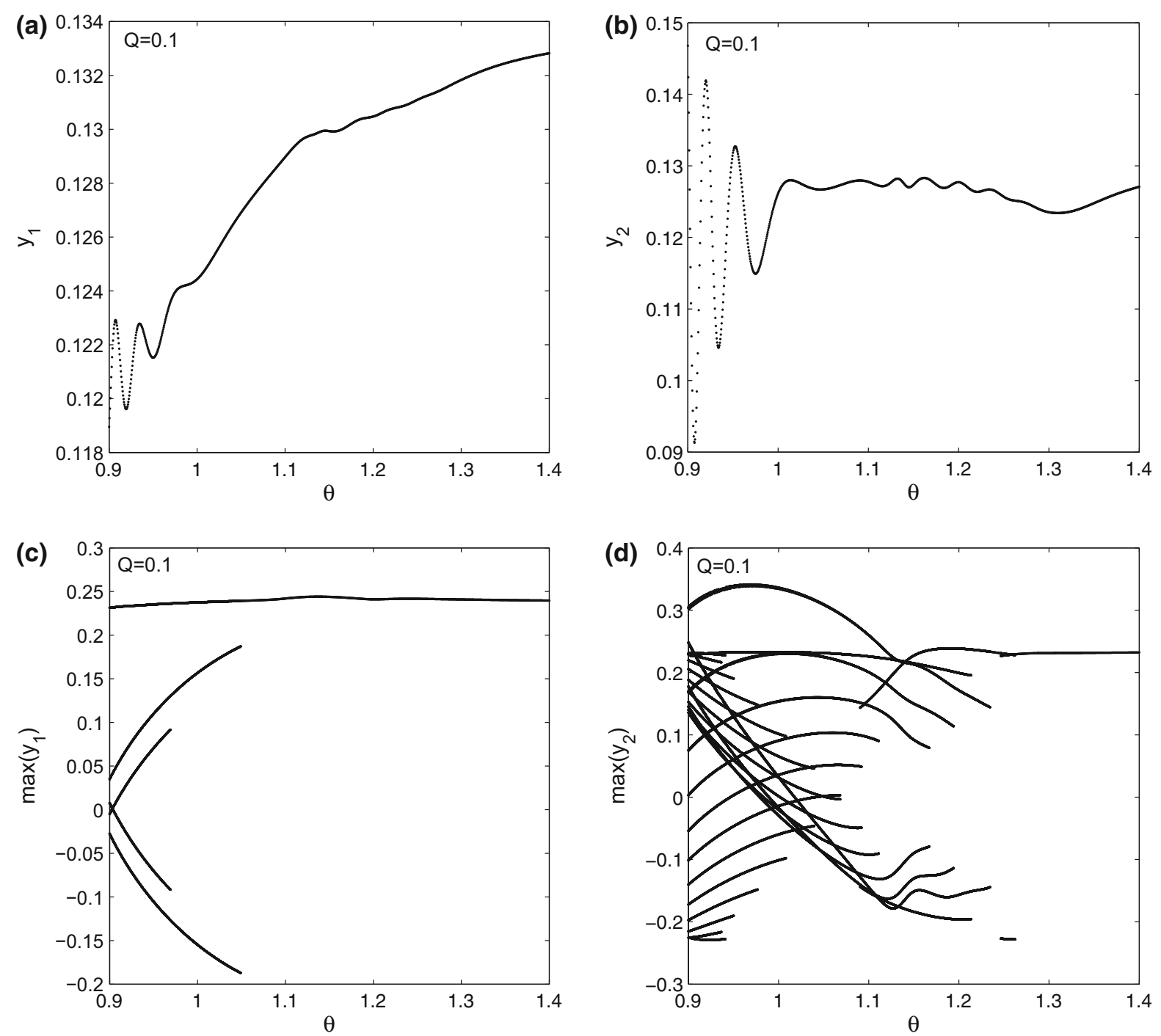

Fig. 19 Classical bifurcation diagrams of the malleus $y_{1}$ (a) and the stapes $y_{2}$ (b) versus temperature $\theta$ at excitation amplitude $Q=0.1$. Maximums of the malleus (c) and the stapes (d) velocity in one period of excitation

temporarily inconvenience can be avoided by a proper selection of SMA which should have lower temperature of austenitic transformation.
Takeing all results into account, it can be concluded the idea of using shape memory alloy to build middle ear prosthesis is promising from practical point of 

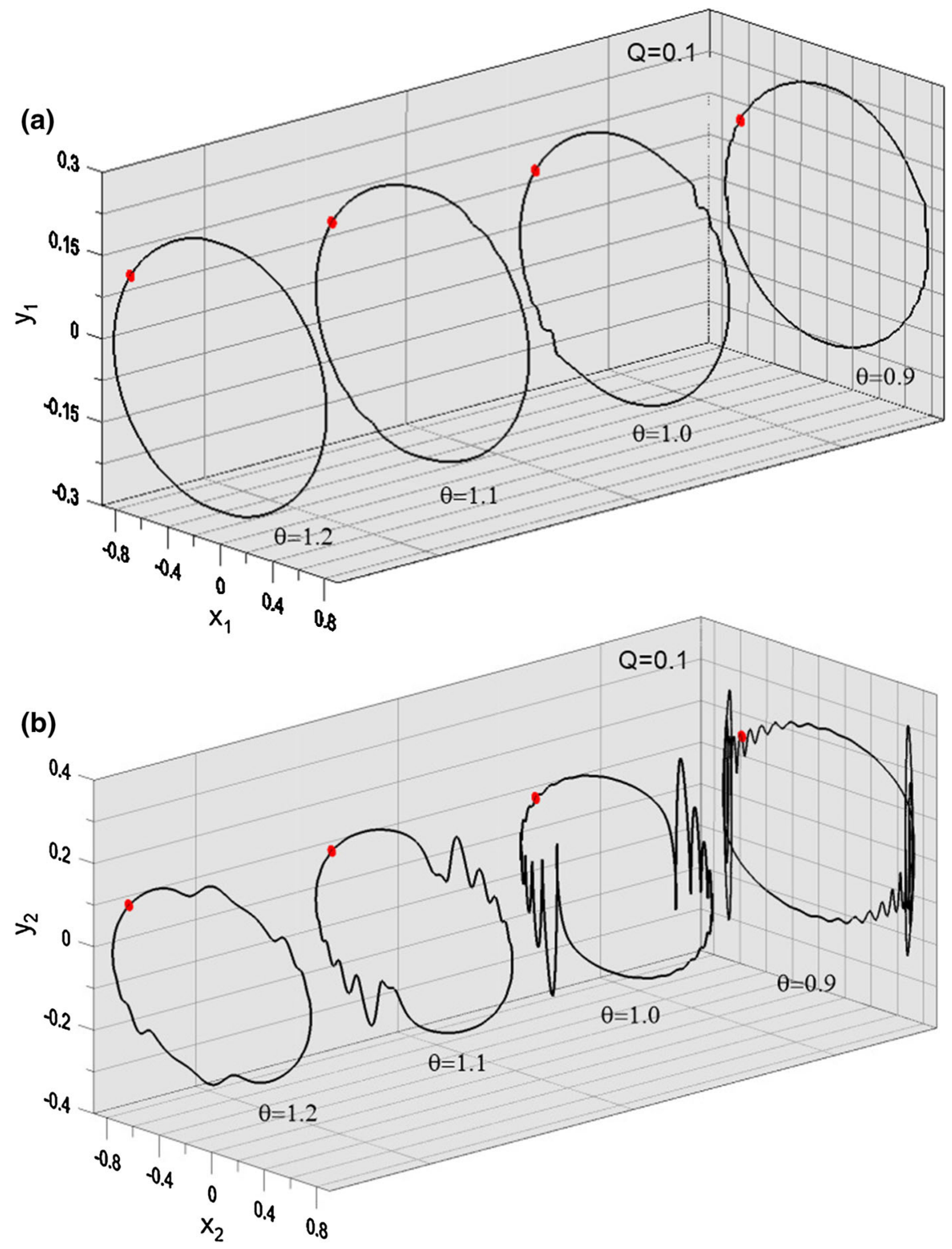

Fig. 20 Change of the malleus (a) and the stapes (b) phase trajectory with temperature $(\theta)$ for $Q=0.1$

view and interesting from scientific point because the analysed system has rich dynamics. In future the authors are planning to made the prosthesis prototype and experimental verification of the results.

Acknowledgements The work is financially supported under the project of National Science Centre (Poland) No. 2014/13/B/ ST8/04047.

Funding This study was funded by National Science Centre (Poland, Grant No. 2014/13/B/ST8/04047).

\section{Compliance with ethical standards}

Conflict of interest The authors declare that they have no conflict of interest.

Open Access This article is distributed under the terms of the Creative Commons Attribution 4.0 International License (http:// creativecommons.org/licenses/by/4.0/), which permits unrestricted use, distribution, and reproduction in any medium, provided you give appropriate credit to the original author(s) and the source, provide a link to the Creative Commons license, and indicate if changes were made. 


\section{References}

1. Bornitz M, Zahnert T, Hardtke HJ, Huttenbrink KB (1999) Identyfication of parameters for the middle ear model. Audiol Neuro Otol 4:163-169

2. Brinson LC (1993) One-dimensional constitutive behavior of shape memory alloys: thermomechanical derivation with non-constant material functions and redefined martensite internal variable. J Intell Mater Syst Struct 4(2):229-242. https://doi.org/10.1177/1045389X9300400213

3. Brown KD, Gantz BJ (2007) Hearing results after stapedotomy with a nitinol piston prosthesis. Arch Otolaryngol Head Neck Surg 133(8):758-762. https://doi.org/10.1001/ archotol.133.8.758

4. Costa DDA, Savi MA (2017) Nonlinear dynamics of an SMA-pendulum system. Nonlinear Dyn 87(3):1617-1627. https://doi.org/10.1007/s11071-016-3137-y

5. Dai C, Cheng T, Wood MW, Gan RZ (2007) Fixation and detachment of superior and anterior malleolar ligaments in human middle ear: experiment and modeling. Hear Res 230(1-2):24-33

6. Doedel E, Keller HB, Kernevez JP (1991) Numerical analysis and control of bifurcation problems (i): bifurcation in finite dimensions. Int J Bifurc Chaos 01(03):493-520. https://doi.org/10.1142/S0218127491000397

7. Eiber A (1999) Mechanical modeling and dynamical bhavior of the human middle ear. Audiol Neuro Otol 4:170-177

8. Falk F (1980) Model free energy, mechanics, and thermodynamics of shape memory alloys. Acta Metall 28(12):1773-1780. https://doi.org/10.1016/0001-6160(80)90030-9

9. Falk F, Konopka P (1990) Three-dimensional Landau theory describing the martensitic phase transformation of shape-memory alloys. J Phys Condens Matter 2(1):61-77. https://doi.org/10.1088/0953-8984/2/1/005

10. Feng B, Gan RZ (2002) A lumped-parameter mechanical model of human ear for sound transmission. In: Proceedings of the second joint Embs-Bmes conference, vols 1-3, pp 267-268

11. Frémond M, Miyazaki S (1996) Shape memory alloys. In: CISM courses and lectures, vol 351. Springer, Wien, New York

12. Gan RZ, Sun Q (2002) Finite element modeling of human ear with external ear canal and middle ear cavity. In: Proceedings of the second joint Embs-Bmes conference, vol 1-3, pp 264-265

13. Gan RZ, Sun QL, Dyer RK, Chang KH, Dormer KJ (2002) Three-dimensional modeling of middle ear biomechanics and its applications. Otol Neurotol 23(3):271-280

14. Huber AM, Schrepfer T, Eiber A (2012) Clinical evaluation of the NiTiBOND stapes prosthesis, an optimized shape memory alloy design. Otol Neurotol 33(2):132-136. https:// doi.org/10.1097/MAO.0b013e31823e28cb

15. Ihrle S, Eiber A, Eberhard P (2015) Experimental investigation of the three dimensional vibration of a small lightweight object. J Sound Vib 334:108-119. https://doi.org/10. 1016/j.jsv.2014.05.024

16. Ihrle S, Lauxmann M, Eiber A, Eberhard P (2013) Nonlinear modelling of the middle ear as an elastic multibody system-applying model order reduction to acousto- structural coupled systems. J Comput Appl Math 246:18-26. https://doi.org/10.1016/j.cam.2012.07.010

17. Jin M, Lee J, Ahn KK (2015) Continuous nonsingular terminal sliding-mode control of shape memory alloy actuators using time delay estimation. IEEE/ASME Trans Mechatron 20(2):899-909. https://doi.org/10.1109/TMECH.2014. 2323897

18. Lauxmann M, Eiber A, Haag F, Ihrle S (2014) Nonlinear stiffness characteristics of the annular ligament. J Acoust Soc Am 136(4):1756-1767. https://doi.org/10.1121/1. 4895696

19. Liang C, Rogers CA (1990) One-dimensional thermomechanical constitutive relations for shape memory materials. J Intell Mater Syst Struct 1(2):207-234. https://doi.org/10. 1177/1045389X9000100205

20. Machado LG (2003) Nonlinear dynamics and chaos in coupled shape memory oscillators. Int J Solids Struct 40:5139-5156

21. Machado LG, Savi MA, Pacheco P (2004) Bifurcations and crises in a shape memory oscillator. Shock Vib 11(2):67-80

22. Moller AR (1961) Network model of the middle ear. J Acoust Soc Am 33(2):168-176

23. Nakajima HH, Ravicz ME, Merchant SN, Peake WT, Rosowski JJ (2005) Experimental ossicular fixations and the middle ears response to sound: evidence for a flexible ossicular chain. Hear Res 204:60-77

24. Paiva A, Savi MA (2006) An overview of constitutive models for shape memory alloys. Math Probl Eng 2006(43):1-31. https://doi.org/10.1155/MPE/2006/56876

25. Paiva A, Savi MA, Braga AMB, Pacheco PM (2005) A constitutive model for shape memory alloys considering tensile-compressive asymmetry and plasticity. Int J Solids Struct 42(11-12):3439-3457. https://doi.org/10.1016/j. ijsolstr.2004.11.006

26. Ravicz ME, Peake WT, Nakajima HH, Merchant SN, Rosowski JJ (2004) Modeling flexibility in the human ossicular chain: comparision to ossicular fixation data. In: Gyo K, Wada H (eds) Middle ear mechanics in research and otology. Word Scientific, Singapore

27. Rusinek R, Szymanski M, Warminski J (2017) Soft impact in a biomechanical system with a shape memory element. J Comput Nonlinear Dyn. https://doi.org/10.1115/1. 4036614

28. Rusinek R, Warminski J, Szymanski M, Kecik K, Kozik K (2017) Dynamics of the middle ear ossicles with an SMA prosthesis. Int J Mech Sci 127:163-175. https://doi.org/10. 1016/j.ijmecsci.2016.10.004

29. Rusinek R, Warminski J, Zadrozniak M, Szymanski M (2013) Nonlinear approach to modelling of otosclerosis in a human middle ear. Differ Equ Dyn Syst 21(1-2):45-57. https://doi.org/10.1007/s12591-012-0122-x

30. Savi MA (1993) Chaotic vibrations of an oscillator with shape memory. J Braz Soc Mech Sci XV:1-20

31. Savi MA, Braga AMB (eds) (1993) Chaotic response of a shape memory oscillstor with internal constraints

32. Savi MA, Braga AMB (1993) Chaotic vibration of an oscillator with shape memory. J Braz Soc Mech Sci Eng 15(1): $1-20$

33. Savi MA, Pacheco PM, Braga AM (2002) Chaos in a shape memory two-bar truss. Int J Non Linear Mech 37(8):1387-1395. https://doi.org/10.1016/S0020-7462(02)00029-X 
34. Savi MA, Pacheco PM (2002) Chaos and hyperchaos in shape memory systems. Int J Bifurc Chaos 12(03):645-657. https://doi.org/10.1142/S0218127402004607

35. Savi Ma, Pereira-Pinto FHI, Viola FM, de AS Paula, Bernardini D, Litak G, Rega G (2017) Using 0-1 test to diagnose chaos on shape memory alloy dynamical systems. Chaos Solitons Fractals 103:307-324. https://doi.org/10. 1016/j.chaos.2017.06.016

36. Savi MA, Sá MA, Paiva A, Pacheco PM (2008) Tensilecompressive asymmetry influence on shape memory alloy system dynamics. Chaos Solitons Fractals 36(4):828-842. https://doi.org/10.1016/j.chaos.2006.09.043

37. Shang ZJ, Wang ZM (2011) Nonlinear forced vibration for shape memory alloy spring oscillator. Adv Mater Res 250-253:3958-3964. scientific.net/AMR.250-253.3958
38. Sun Q, Gan RZ, Chang KH, Dormer KJ (2002) Computerintegrated finite element modeling of human middle ear. Biomech Modeling Mechanobiol 1(2):109-122

39. Sun QL, Chang KH, Dormer KJ, Dyer RK, Gan RZ (2002) An advanced computer-aided geometric modeling and fabrication method for human middle ear. Med Eng Phys 24(9):595-606

40. Tanaka KO, Nagaki SO (1982) A thermomechanical description of materials with internal variables in the process of phase transitions. Ing Arch 51:287-299

41. Taschke H, Weistenhofer C, Hudde H (2000) A full-size physical model of the human middle ear. Acustica 86(1):103-116

42. Zwislocki J (1962) Analysis of the middle-ear function. Part I: input impedance. J Acoust Soc Am 34(8):1514-1523 